\begin{tabular}{|c|l|}
\hline Title & $\begin{array}{l}\text { A LEVEL SET A PPROA CH REFLECTING SHEET ST RUCTURE WITH SINGLE A UXILIARY FUNCTION FOR } \\
\text { EV OLVING SPIRA LS ON CRY STA L SURFACES }\end{array}$ \\
\hline Author(s) & Ohtsuka, Takeshi; Tsai, Y en-Hsi R.; Giga, Yoshikazu \\
\hline Citation & Hokkaido University Preprint Series in Mathematics, 1025, 1-36 \\
\hline Issue Date & 2012-12-16 \\
\hline DOI & 10.14943/84171 \\
\hline Doc URL & http:/hdl.handle.net/2115/69830 \\
\hline Type & bulletin (article) \\
\hline File Information & pre1025.pdf \\
\hline
\end{tabular}

Instructions for use 


\title{
A LEVEL SET APPROACH REFLECTING SHEET STRUCTURE WITH SINGLE AUXILIARY FUNCTION FOR EVOLVING SPIRALS ON CRYSTAL SURFACES
}

\author{
TAKESHI OHTSUKA, YEN-HSI RICHARD TSAI, AND YOSHIKAZU GIGA \\ In memory of Professor Rentaro Agemi
}

\begin{abstract}
We introduce a new level set method to simulate motion of spirals in a crystal surface governed by an eikonal-curvature flow equation. Our formulation allows collision of several spirals and different strength (different modulus of Burgers vectors) of screw dislocation centers. We represent a set of spirals by a level set of a single auxiliary function $u$ minus a pre-determined multi-valued sheet structure function $\theta$, which reflects the strength of spirals (screw dislocation centers). The level set equation used in our method for $u-\theta$ is the same as that of the eikonal-curvature flow equation.

The multi-valued nature of the sheet structure function is only invoked when preparing the initial auxiliary function, which is nontrivial, and in the final step when extracting information such as the height of the spiral steps. Our simulation enables us not only to reproduce all speculations on spirals in a classical paper by Burton, Cabrera and Frank (1951) but also to find several new phenomena.
\end{abstract}

\section{INTRODUCTION}

Consistent spiral patterns are observed in many crystal growth situations. The center of a spiral is believed to be the location where a screw dislocation in a crystal lattice terminates on the crystal surface, while the spiral being a step (discontinuity) in the crystal height. Atoms bond with the crystal structure with a higher probability near a step and thus results in an evolution of the step. The dynamics of the step in this setting is well studied and traces back to Burton, Cabrera and Frank [BCF51].

Consider a spiral pattern drawn by steps on a growing crystal surface. In the theory of the crystal growth in [BCF51], steps evolve with a normal velocity of the form

$$
V=C-\kappa,
$$

where $C$ is a constant denoting a driving force, and $\kappa$ is the curvature of the curve drawn by the steps. The equation (1.1) is sometimes called an eikonal-curvature flow equation. In [BCF51] the equation (1.1) is given as $V=v_{\infty}\left(1-\rho_{c} \kappa\right)$ with the velocity of straight

2010 Mathematics Subject Classification. 53C44; 35K65; 65L12.

Key words and phrases. Evolution of spirals; Level set method; Sheet structure function; Eikonal and curvature flow; Finite difference scheme.

The work of the first author is partly supported by the Japan Society for the Promotion of Science (JSPS) through Grant-in-Aid for Young Scientists (B)22740109.

The work of the second author is partly supported by NSF grants DMS-1217203, DMS-0914465, and DMS-0914840.

The work of the third author is partly supported by JSPS grants Kiban(S)21224001 and Kiban(A)23244015. 
line steps $v_{\infty}$ and the critical radius $\rho_{c}$ for the generation of two dimensional kernel from supersaturation. The curvature term, $\kappa$, is interpreted as a result of the GibbsThompson effect. The sign of curvature is taken so that (1.1) is a parabolic equation. Our formulation, however, includes the case of the negative driving force, i.e., when a crystal is melting.

The spiral crystal growth problem can be studied by direct numerical simulation using a variety of techniques. A straight forward approach is to track the spiral by putting a set of markers on the spiral and solve the resulting system of ODEs that determine the marker locations in time. It is also possible to use Monte-Carlo type algorithms for simulations of small domains.

Since the spiral dynamics generally involve merging of different spirals, implicit interface methods can be of an attractive option. A phase field model was introduced in [KP98] or [Kob10] for spiral growth simulations. This is a diffuse interface method that requires fine grid resolution at least in a neighborhood of the evolving spirals. Conventional level set methods [OS88] [Set99, OF01] (see for its foundation in mathematical analysis [Gig06]) do not apply directly; in a typical level set method involving a Lipschitz function, $u$, as the so-called level set function, the point set $\{x ; u(t, x)=0\}$ corresponds to a curve which divides the domain into two disjoint sets (the typical example is a closed curve by itself or combining it and the boundary of the domain). However, a spiral generally does not divide the domain into two disjoint sets. In [Sme00] Smereka introduced a level set formulation to simulate a spiral crystal growth numerically. This is an interesting and pioneering work simulation of evolving spirals. In his formulation a spiral is described by two continuous auxiliary functions (level set functions), and the intersection of the zero level sets of these two functions represents the spiral center (screw dislocation). The dynamics of the spiral is computed by solving two partial differential equations (PDEs) that contain discontinuous coefficients. The height function is computed by solving a Poission equation with a Dirac- $\delta$ source concentrated along the spiral.

While the level set method in [Sme00] is powerful to study collision of several spirals, it does not apply when two spiral centers have different strengths -a case in which the crystal surface includes several screw dislocations with Burgers vectors of different magnitudes. In [Oht03] the first author introduced a new level set method using only one auxiliary function but using a sheet structure function introduced by Kobayashi [Kob10] in 1990. The sheet structure function reflects a helical structure formed by ordered atoms in a crystal, and thus this method enables us to describe more general situation including multiple centers with different strengths. While the analytic foundation for this method in [Oht03] based on viscosity solutions [Oht03] [GNO08] is well-established, numerical simulation based on this idea was not yet studied or published; among many computational issues, the construction of initial auxiliary function is not trivial.

In this paper, we propose an algorithm for computing evolving spirals by (1.1) based on the level set method using a sheet structure function. Our method does compute correctly the behavior of co-rotating spirals and spirals with different rotational orientations with possibly different strengths. We recover all speculations for spirals given by [BCF51] in our numerical simulations. We also find several new phenomena.

Let us recall the level set method in [Oht03]. A crystal surface is to be described in a bounded domain $\Omega$ in the plane. A screw dislocation center is assumed to be a 
closed ball of finite size in our formulation. We consider $N$ (disjoint) screw dislocations $\bar{U}_{1}, \bar{U}_{2}, \ldots, \bar{U}_{N}$ whose centers are denoted by $a_{1}, a_{2}, \ldots, a_{N}$, on the surface. A spiral $\Gamma_{t}$ at time $t \geq 0$ lies on a domain $W$ which is obtained from $\Omega$ by removing all screw dislocation centers, i.e., $W=\Omega \backslash \bigcup_{j=1}^{N} \overline{U_{j}}$. In this paper we assume that the end points of $\Gamma_{t}$ always stay on the boundary $\partial W$ of $W$ with the orthogonality condition,

$$
\Gamma_{t} \perp \partial W .
$$

Thus, while $\Gamma_{t}$ is not a closed curve, its image is a relatively closed point set in $\bar{W}$. We now introduce a sheet structure function $\theta$, which is due to Kobayashi [Kob10],

$$
\theta(x)=\sum_{j=1}^{N} m_{j} \arg \left(x-a_{j}\right)
$$

with non-zero integers $m_{1}, \ldots, m_{N}$, where $m_{j}$ is taken so that $z=\theta(x)$ gives the helical structure. The constant $m_{j}$ quantifies the strength of the spiral center $a_{j}$. Thus, the level set formulation of $\Gamma_{t}$ in [Oht03] is given by

$$
\Gamma_{t}=\{x \in \bar{W} ; u(t, x)-\theta(x)=0 \text { with modulo } 2 \pi\} .
$$

In this formulation spirals are given by the cross-section between an auxiliary cone described by $u(t, x)$ and a helical surface $z=\theta(x)$. With this formulation we derive a level set equation corresponding to motion of spirals by (1.1). Moreover, we construct a surface height function from a solution of the level set equation.

While the existence of the initial data $u_{0}$ for a given initial spirals $\Gamma_{0}$ was established in [GNO08], construction of initial auxiliary function $u_{0}$ at practical level is still difficult, because the method requires one to take a branch of sheet structure functions whose discontinuity is only on $\Gamma_{0}$. To overcome this difficulty we first give a practical way to construct $u_{0}$ for a spiral with a single dislocation center, and then for a line spiral connecting between two centers. We further give an additive way to construct an initial auxiliary function $u_{0}$ inductively with respect to numbers of screw dislocations. We also present a way to construct $u_{0}$ for a complicated spiral with a single center.

A crucial advantage of our method is the use of a single scalar equation, even for situations involving multiple centers with different strengths. In particular, our singleequation formulation is useful when considering evolution of several spirals associated with one screw dislocation. With our method, it suffices to choose a suitable coefficient in front of the argument function arg, whose origin is the screw dislocation center in our method. Our single-equation formulation also enables us to compare the activities between a group of screw dislocations with co-rotating single spirals and one screw dislocations with multiple spirals. Smereka in [Sme00] treats a pair of co-rotating spirals or those with opposite rotational orientations when the pair is far apart, i.e, the distance of the pair is larger than $2 \pi / C$ in the evolution by (1.1), which is the critical distance proposed by [BCF51]. Our method is able to examine not only a close pair of spirals but also a group of several (of course two or more) screw dislocations.

In the paper, on the one hand we numerically verify all speculations for spirals given by [BCF51], on the other hand we examine some situations that are not discussed in [BCF51]. While Burton et al discussed the activity of a group of screw dislocations, they did not discuss the situation in which screw dislocation centers with different strengths 
co-exist on the surface. In this paper we demonstrate simulations involving configurations such as a pair of co-rotating or opposite oriented spirals, and several screw dislocations with different rotational orientations and strengths. Anisotropic motion is not treated in this paper, but our formulation also can apply to the anisotropic evolution with a smooth and strictly convex surface energy density; see [Gig06] for detail for a formulation of an anisotropic evolution.

Nevertheless, there remain some situations to which our method do not apply. While [Sme00] and this paper study the dynamics of the spirals formed by steps centering at a set of dislocations, the dynamics of the screw dislocations (including screw dislocation centers) in the crystals are not modeled. In [XCSE03] and [XSCW04], Xiang et. al. proposed another level set formulation to compute the motion of screw dislocation in crystals. In their level set formulation, screw dislocations are implicitly represented as the intersection of two level set functions defined in three dimensions. One of the further difficulties for modeling the dynamics of screw dislocations in our method resides in the need to remove neighborhoods of screw dislocations from the surface (in numerical computations it suffices to remove one grid point when a screw dislocation center is on the grid point). However, if the screw dislocation center is just a single point, theoretical treatment seems to be difficult. For this direction there is a work by Forcadel, Imbert and Monneau [FIM] but their setting is somewhat restrictive. Imai, Ishimura and Ushijima [IIU99] presented a formulation of an evolving spiral by crystalline curvature flow with no driving force and gave some numerical simulations as well as a proof for local well-posedness.

Several interesting results on existence and behavior of spirals are obtained by approaches based on ordinary and partial differential equations, shortly (ODE) and (PDE). In an ODE approach several interesting self-similar spiral type solutions are constructed and classified in various settings (e.g. [Ish98], [FGT04], [FGT06], [GNOT06]). In a PDE approach, several results on Lyapunov or asymptotic stability of rotating spirals are derived; see e.g. [GIK02]. Ogiwara and Nakamura [ON03] studied a diffuse interface model proposed by Kobayashi [Kob10], and established the existence and asymptotic stability of steadily rotating spirals. In particular, their stability result implies that, when we consider the evolution of $m$ spirals associated with one center, then the spiral pattern with $1 / m$ times rotation symmetry is asymptotic stable. This result is different from the behavior with similar situation in our method. This phenomenon will be discussed in detail in our forthcoming paper [GOT].

This paper is organized as follow: In the following subsection, we present the idea of using a sheet structure function to define a spiral; in Section 2 we present our proposed level set formulation for the simulation of spiral crystal growth. In Section 3, we present some numerical simulations involving spirals of different configurations.

\section{A LEVEL SET FORMULATION USING SHEET STRUCTURES}

2.1. Spirals on a plane. We consider a growing crystal surface with $N(\geq 1)$ screw dislocations over a bounded domain $\Omega \subset \mathbb{R}^{2}$. Screw dislocations typically result in discontinuities in the crystal height that connects to the dislocations. In this paper, these discontinuities are called steps in the crystal height. The location of the steps are spiral curves which we will model and evolve, and in later parts of the paper, we will use 'curves' and 'steps' interchangeably in this paper. 
Associated with the screw dislocations are the centers of spirals, denoted by $a_{1}, a_{2}, \ldots, a_{N}$, which are assumed to be stationary. For a technical reason we further assume that a (screw dislocation) center consists a neighborhood $U_{j}$ of $a_{j}$, and $\bar{U}_{i} \cap \bar{U}_{j}=\emptyset$ for $i \neq j$. We remove all $\bar{U}_{j}$ from $\Omega$, and thus set $W=\Omega \backslash\left(\bigcup_{j=1}^{N} \bar{U}_{j}\right)$. On this domain, spirals can be defined by parameterized curves

$$
\Gamma:=\left\{P(s) \in \bar{W} ; s \in\left[0, s_{0}\right]\right\} .
$$

As we shall see later, the height of the crystal surface can be defined from the configuration of spirals.

In this paper, we consider evolving spirals $\Gamma_{t}$ in $\bar{W}$. To guarantee the unique solvability of the initial value problem for (1.1) we impose the right angle boundary condition (1.2) on $\partial W$ (see [Oht03] [GNO08]).

As in [GNO08] it is convenient to classify spirals into two types - a simple spiral and a connecting spiral - depending on the feature whether or not it touches the boundary $\partial \Omega$ of the crystal surface $\Omega$.

Definition 2.1. Let $\Gamma$ be given by $(2.1)$.

(i) For a given point $a \in \Omega$ let $U$ be a neighborhood of $a$ satisfying $U \subset \Omega$ whose boundary does not touch $\partial \Omega$, and $W=\Omega \backslash \bar{U}$. We say $\Gamma$ is a $C^{n}(n \in \mathbb{N} \cup\{0\})$ simple spiral associated with $a \in \Omega$ if

(S1) $P(s) \in C^{n}\left(\left[0, s_{0}\right]\right)$ and $|\dot{P}(s)| \neq 0$ for $s \in\left[0, s_{0}\right]$ if $n \geq 1$, where $\dot{P}=d P / d s$,

(S2) $P(0) \in \partial U, P\left(s_{0}\right) \in \partial \Omega$ and $P(s) \notin \partial W$ for $s \in\left(0, s_{0}\right)$ holds.

(ii) For given points $a_{1}, a_{2} \in \Omega$ let $U_{1}$ and $U_{2}$ be neighborhoods of $a_{1}$ and $a_{2}$ respectively, and $W=\Omega \backslash \overline{U_{1} \cup U_{2}}$. Assume that $\bar{U}_{1}$ and $\bar{U}_{2}$ is disjoint, i.e., $\bar{U}_{1} \cap \bar{U}_{2}=\emptyset$, and $U_{i} \subset \Omega$ whose boundary does not touch $\partial \Omega$ for $i=1,2$. We say $\Gamma$ is a $C^{n}$ connecting spiral between $a_{1}$ and $a_{2}$ (or associated with $a_{1}$ and $a_{2}$ ) if (S1) and $\left(\mathrm{S} 2^{\prime}\right) P(0) \in \partial U_{1}, P\left(s_{0}\right) \in \partial U_{2}$, and $P(s) \notin \partial W$ for $s \in\left(0, s_{0}\right)$ holds.

For the case $\left.W=\Omega \backslash\left(\bigcup_{i=1}^{N} \bar{U}_{i}\right)\right)$ with (mutually disjoint) neighborhoods $U_{i}$ of $a_{i}$ for $i=1, \ldots, N$, we call a connecting spiral between $a_{i}$ and $a_{j}$ simply an $(i, j)$ connecting spiral for simplicity.

Remark 2.2. Note that an $(i, j)$ connecting spiral is also a $(j, i)$ connecting spiral by taking $Q(s)=P\left(s_{0}-s\right)$. However, we ignore the direction of the connection in the following arguments.

Spirals on a plane have two orientations, one is related to the evolution and the other to rotation with respect to a screw dislocation center. The orientation of the evolution is defined as a continuous unit normal vector field on the curve, we denote this vector field by $\mathbf{n}$. The orientation of the rotation can be defined by the relation between the tangent and the normal vectors of the spiral as in Definition 2.3. These orientations should not be confused with rotations of the self-similar spiral structure resulted from the spiral evolution. 
Definition 2.3. Let $\Gamma$ be a $C^{1}$ simple or connecting spiral associated with $a \in \Omega$ at $P(0)$. Let $s$ in $P(s)$ be an arclength parameter. We say that $\Gamma$ has a counter-clockwise (resp. clockwise) orientation with respect to $a \in \Omega$ if

$$
\mathbf{n}(P(s))=\left(\begin{array}{cc}
0 & -1 \\
1 & 0
\end{array}\right) \dot{P}(s) \quad\left(\text { resp. }-\left(\begin{array}{cc}
0 & -1 \\
1 & 0
\end{array}\right) \dot{P}(s)\right)
$$

holds for $s \in\left[0, s_{0}\right]$.
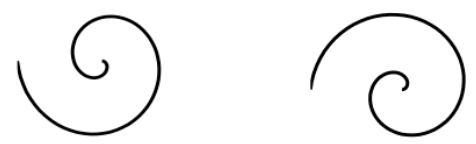

Figure 2.1. Two spirals with opposite rotational orientations. The one on the left has a counter-clockwise orientation.

Figure 2.1 depicts two spirals of opposite rotational orientations.

Remark 2.4. If an $(i, j)$ connecting spiral has a counter-clockwise orientation w.r.t. $a_{i}$, then it has a clockwise orientation w.r.t. $a_{j}$. In fact, we set $Q(s)=P\left(s_{0}-s\right)$ to obtain

$$
\mathbf{n}(Q(s))=\mathbf{n}\left(P\left(s_{0}-s\right)\right)=\left(\begin{array}{cc}
0 & -1 \\
1 & 0
\end{array}\right) \dot{P}\left(s_{0}-s\right)=-\left(\begin{array}{cc}
0 & -1 \\
1 & 0
\end{array}\right) \dot{Q}(s)
$$

for $s \in\left[0, s_{0}\right]$. Moreover, one finds that the rotational orientations for connecting spirals is uniquely determined in spite of the direction $(i, j)$ or $(j, i)$ of the connection.

We now define the generalized number of spirals associated with a center.

Definition 2.5. Let $a_{i} \in \Omega$ be a center for $i=1, \ldots, N$. We define the signed number of spirals associated with $a_{i}$ as

$$
m_{i}=m_{i}^{+}-m_{i}^{-},
$$

where $m_{i}^{+}$and $m_{i}^{-}$are respectively the number of spirals which are associated with $a_{i}$ and which have counter-clockwise and clockwise orientations.

Physically speaking in our setting the Burgers vector is orthogonal to the plain containing $\Omega$ and its modulus equals $\left|m_{i}\right|$. We shall exclude the case $m_{i}=0$. 


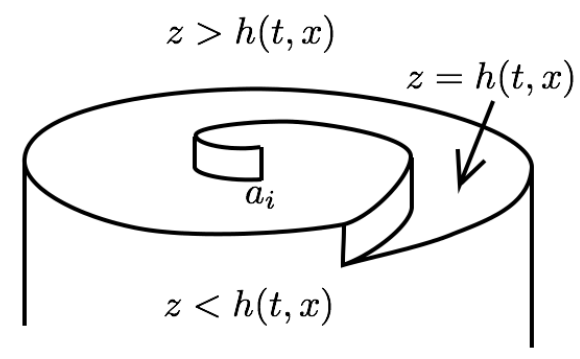

FiguRE 2.2. Surface and the height function.

2.2. The proposed level set formulation. For simplicity we consider a counterclockwise oriented spiral associated with the origin. When the driving force, $\mathrm{C}$ in (1.1), is positive, this spiral moves around the origin counter-clockwise, and the corresponding crystal surface grows up with the climbing steps on a helical surface similar to a spiral staircase. We interpret this phenomena as step evolution on the Riemann surface " $z=$ $\arg x "$. In this case, writing the crystal surface as a height function $h(t, x)$, we can define interior and exterior sets by $\{(x, z) \in \bar{W} \times \mathbb{R} ; z<h(t, x)\}$ and $\{(x, z) \in \bar{W} \times \mathbb{R} ; z>$ $h(t, x)\}$, respectively. However, it suffices to consider the height $z$ only on the helical surface " $z=\arg x$ " in our case. To complete our idea rigorously, we introduce a covering space as in [Oht03], which is

$$
\mathfrak{X}:=\left\{(x, \xi) \in \bar{W} \times \mathbb{R}^{N} ;\left(\cos \xi_{i}, \sin \xi_{i}\right)=\left(x-a_{i}\right) /\left|x-a_{i}\right| \text { for } i=1, \ldots, N\right\},
$$

where $\xi=\left(\xi_{1}, \ldots, \xi_{N}\right)$. If $N=1$, the set $\mathfrak{X}$ corresponds to the surface $z=\arg x$.

Definition 2.6. Let $m_{i} \in \mathbb{Z} \backslash\{0\}$ be the signed number of spirals associated with $a_{i}$. We say $\widetilde{\Gamma}$ is a generalized spiral curve on $\mathfrak{X}$ if there exists $u \in C(\bar{W})$ satisfying

$$
\widetilde{\Gamma}=\left\{(x, \xi) \in \mathfrak{X} ; u(x)-\sum_{i=1}^{N} m_{i} \xi_{i}=0\right\}
$$

Moreover, we call

$$
\begin{aligned}
& \widetilde{I}:=\left\{(x, \xi) \in \mathfrak{X} ; u(x)-\sum_{i=1}^{N} m_{i} \xi_{i}>0\right\}, \\
& \widetilde{O}:=\left\{(x, \xi) \in \mathfrak{X} ; u(x)-\sum_{i=1}^{N} m_{i} \xi_{i}<0\right\}
\end{aligned}
$$

respectively the interior and exterior sets of $\widetilde{\Gamma}$.

In the evolution of spirals on the plane, the division of interior and exterior makes sense only locally. It is inconvenient for the level set method, in particular to determine the direction of the evolution. The covering space we introduced enables us to determine the interior and exterior globally in the space. In particular, the inequality in the definition of interior is related to the Figure 2.2; the term $\sum_{j=1}^{N} m_{j} \xi_{j}$ means the height in the covering 
space and thus the inequality means $z<u(x)$ like as the Figure 2.2, which implies that $u$ plays the role of the height function.

We now give a level set formulation for evolving spirals from the above configuration. Let $\Gamma_{t}$ be a given evolving spiral on $\bar{W}$ with orientation of evolution $\mathbf{n}$ at time $t$ and with a signed numbers $m_{j}$ associated with $a_{j}$. We describe it as

$$
\Gamma_{t}=\{x \in \bar{W} ; u(t, x)-\theta(x) \equiv 0 \quad \bmod 2 \pi \mathbb{Z}\}, \quad \mathbf{n}=-\frac{\nabla(u-\theta)}{|\nabla(u-\theta)|}
$$

with an auxiliary function $u:[0, T] \times \bar{W} \rightarrow \mathbb{R}$ and a sheet structure function

$$
\theta(x) \equiv \sum_{i=1}^{L} m_{i} \arg \left(x-a_{i}\right)
$$

Naturally, in this formulation for spirals, $\theta$ has to be multiple-valued. We can choose other multiple-valued function whose derivative are uniquely defined in spite of choices of branches. However, our choice of $\theta$ as of the form (2.3) is physically important because it helps describe the height of the crystal surface; see Section 2.5 for detail.

Once we obtain $u$ by solving the evolution equation corresponding (1.1)-(1.2), which is (2.4)-(2.6) in $\S 2.3$, we can extract the evolving spirals by (2.2). However, for numerical purposes, we need to remove the spurious zero level set that results from the branch cuts used in defining $\theta$.

Without loss of generality, we assume that $a_{1}^{1}<a_{2}^{1}<\cdots<a_{N}^{1}$ for $a_{j}=\left(a_{j}^{1}, a_{j}^{2}\right)$, $j=1, \ldots, N$. We decompose $\bar{W}$ into the union of vertical strips, separated by the centers and extract $\Gamma$ in each strip. We set

$$
W_{j}= \begin{cases}\left\{x=\left(x^{1}, x^{2}\right) \in \bar{W} ; x^{1} \leq a_{1}^{1}\right\} & \text { if } j=0, \\ \left\{x=\left(x^{1}, x^{2}\right) \in \bar{W} ; a_{j}^{1} \leq x^{1} \leq a_{j+1}^{1}\right\} & \text { if } j=1, \ldots, N-1, \\ \left\{x=\left(x^{1}, x^{2}\right) \in \bar{W} ; x^{1} \geq a_{N}^{1}\right\} & \text { if } j=N .\end{cases}
$$

Let $\widehat{\Theta}_{j}^{-}: \bigcup_{i=j}^{N} W_{i} \rightarrow(-\pi, \pi]$ and $\widehat{\Theta}_{j}^{+}: \bigcup_{i=0}^{j-1} W_{i} \rightarrow(0,2 \pi]$ be the corresponding smooth branches of $\arg \left(x-a_{j}\right)$, and define $\widehat{\Theta}_{j}: W_{j} \rightarrow \mathbb{R}$ by

$$
\widehat{\Theta}_{j}(x)=\sum_{i=1}^{j} m_{i} \widehat{\Theta}_{i}^{-}(x)+\sum_{i=j+1}^{N} m_{i} \widehat{\Theta}_{i}^{+}(x) \quad \text { for } j=0, \ldots, N .
$$

We here note that $\sum_{i=1}^{0} m_{i} \widehat{\Theta}_{i}^{-}(x)=\sum_{i=N+1}^{N} m_{i} \widehat{\Theta}_{i}^{+}(x) \equiv 0$. Hence, $\widehat{\Theta}_{j}$ is smooth in $W_{j}$ (see Figure 2.2), and the spiral $\Gamma_{t}$ can be unambiguously defined by

$$
\Gamma_{t}=\bigcup_{j=0}^{N}\left(\Gamma_{t} \cap W_{j}\right)=\bigcup_{j=0}^{N} \bigcup_{k=-\hat{k}_{j}}^{\hat{k}_{j}}\left\{x \in W_{j} ; u(t, x)-\widehat{\Theta}_{j}(x)=2 \pi k\right\},
$$

where $\hat{k}_{j}$ is the smallest integer satisfying $\max _{W_{j}}\left|u(t, \cdot)-\widehat{\Theta}_{j}\right|<2 \pi \hat{k}_{j}$. 


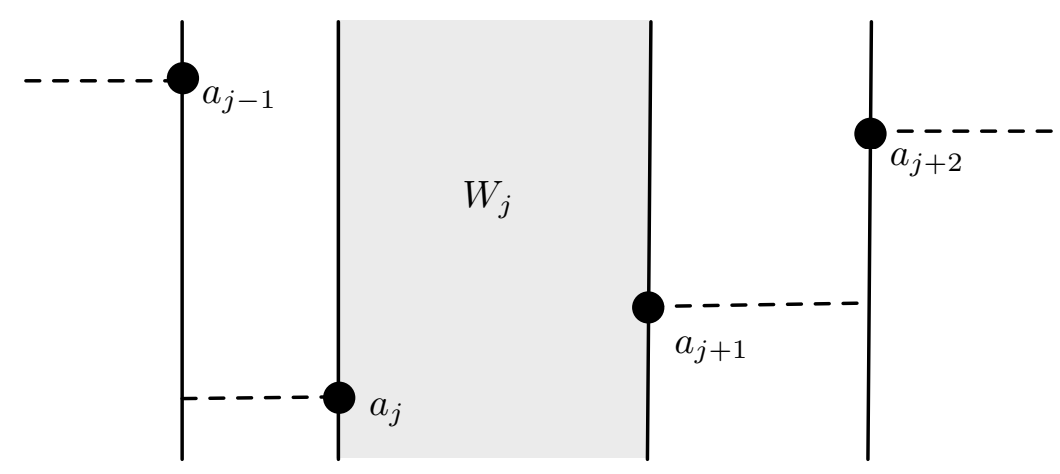

FiguRE 2.3. Branch cuts of $\widehat{\Theta}_{j}$.

2.3. Dynamics. Although our formulation (2.2) includes a multi-valued function $\theta$, it is essentially the same as a level set formulation by a smooth branch of $w=u-\theta$ locally. Thus we have

$$
\mathbf{n}=-\frac{\nabla(u-\theta)}{|\nabla(u-\theta)|}, V=\frac{u_{t}}{|\nabla(u-\theta)|}, \kappa=-\operatorname{div} \frac{\nabla(u-\theta)}{|\nabla(u-\theta)|} .
$$

The equations (1.1) and (1.2) are represented as follows (see [Gig06] for details);

$$
\begin{aligned}
u_{t}-|\nabla(u-\theta)|\left\{\operatorname{div} \frac{\nabla(u-\theta)}{|\nabla(u-\theta)|}+C\right\} & =0 \quad \text { in } \quad(0, T) \times W, \\
\langle\vec{\nu}, \nabla(u-\theta)\rangle & =0 \text { on } \quad(0, T) \times \partial W .
\end{aligned}
$$

Precisely speaking, the system (1.1)-(1.2) is formally equivalent to (2.4)-(2.5) only on spirals. The main idea of a level set method is to consider the system (2.4)-(2.5) not only on spirals but also on whole $W$.

For a simulation of the evolution we choose $u_{0} \in C(\bar{W})$ satisfying

$$
\Gamma_{0}=\left\{x \in \bar{W} ; u_{0}(x)-\theta(x) \equiv 0 \quad \bmod 2 \pi \mathbb{Z}\right\}
$$

for a given initial curve $\Gamma_{0}$, and solve the initial-boundary value problem (2.4), (2.5) and

$$
\left.u\right|_{t=0}=u_{0}
$$

to describe evolutions of spirals.

Much analysis of (2.4)-(2.5) has been done; the mathematical framework of our proposed approach is complete. In [Oht03] the first author established a comparison principle for viscosity solutions of (2.4)-(2.5), which implies the uniqueness of solutions, and the existence of a time-global solution for a continuous initial datum $u_{0}$. Goto, Nakagawa and the first author [GNO08] obtained the comparison principle of interior and exterior sets on $\mathfrak{X}$, and thus the uniqueness of level sets $\Gamma_{t}$ with respect to an initial curve $\Gamma_{0}$ is established. They also construct a continuous initial data $u_{0}$ such that (2.2) holds for a given $\Gamma_{0}$. Note that it is nontrivial to construct a suitable auxiliary function $u_{0}$ for a given initial spiral $\Gamma_{0}$ which is quite different from conventional level set approach [ES91], [CGG91], [Gig06]. Furthermore, it is rather easy to see [CIL92], [CGG91], [ES91], [Gig06] 
that the viscosity solutions of the regularized problem

$$
u_{t}-|\nabla(u-\theta)|\left\{\operatorname{div} \frac{\nabla(u-\theta)}{\sqrt{\varepsilon^{2}+|\nabla(u-\theta)|^{2}}}+C\right\}=0
$$

converges locally uniformly to the viscosity solution of (2.4)-(2.5). In a later section we present numerical simulations based on (2.7).

2.4. Initialization. For a given bunch of spirals $\Gamma$ it is nontrivial to find $u \in C(\bar{W})$ satisfying

$$
\Gamma=\{x \in \bar{W} ; u(x)-\theta(x) \equiv 0 \quad \bmod 2 \pi \mathbb{Z}\} .
$$

Goto, Nakagawa and the first author show in [GNO08] the existence of $u \in C(\bar{W})$ satisfying (2.8). However, their method is difficult to carry out in practical level. In fact, they first construct $\theta_{\Gamma}$ which is a smooth branch of $\theta$ with branch-cut line on $\Gamma$. Next, they mollify it with linear interpolation in very thin tubular neighborhood around of $\Gamma$. Thus, the difficulties lie in the construction of $\theta_{\Gamma}$ and the choice of tubular neighborhood. In particular, the second step is crucial since that the width of neighborhood depends on the size of removed neighborhoods around $a_{j}$. In fact, the method of [GNO08] would construct initial data with $|\nabla(u-\theta)|=O\left(\Delta x^{-1}\right)$ if the diameter of removed neighborhood is $O(\Delta x)$, where $\Delta x$ is a spatial lattice span.

In this subsection, we shall give a practical way to construct smoother $u$ for a class of simple spirals centering at the origin. Next, we give an additive way of constructing $u$ from those of simpler spirals. In particular, we shall give a practical way to construct $u$ for any initial configuration whose curve segmentations consist of straight lines. Furthermore, we shall consider here only the case for a single simple spiral with counter-clockwise orientation with respect to the origin, i.e. when $\theta(x)=\arg x$ since the data $v$ for $\Gamma$ with $\theta(x)=-\arg x$ is given by $v=-\tilde{u}$ which is the data for $\left\{\left(x^{1},-x^{2}\right) \in \bar{W} ;\left(x^{1}, x^{2}\right) \in \Gamma\right\}$.

Spreading spiral associated with the origin. Let $\Gamma$ be given by

$$
\Gamma=\{r(\cos \xi(r), \sin \xi(r)) \in \bar{W} ; r \in[\rho, R]\}
$$

with a continuous function $\xi \in C([\rho, R])$. In this case we set $u$ as

$$
u(x):=\xi(|x|) .
$$

In particular, a line $\{r(\cos \alpha, \sin \alpha) ; r \in[\rho, R]\}$ for an angle constant $\alpha$ is given by $u(x)=\alpha$.

Connecting straight line between two centers. The above idea for a line enables us to find that

$$
u(x)=\pi \quad \text { for } x \in \bar{W}
$$

gives a connecting line

$$
\Gamma=\left\{\sigma a_{1}+(1-\sigma) a_{2} \in \bar{W} ; \sigma \in[0,1]\right\}
$$

between two centers $a_{1}, a_{2} \in \Omega$. In fact, let $L=\left\{\sigma a_{1}+(1-\sigma) a_{2} \in \mathbb{R}^{2} ; \sigma \in \mathbb{R}\right\}=\{x \in$ $\left.\mathbb{R}^{2} ;\left(x-a_{1}\right) \cdot p_{L}=0\right\}$, where $p_{L} \in S^{1}$ satisfying $p_{L} \cdot\left(a_{2}-a_{1}\right)=0$. Set

$$
W_{1}=\left\{x \in \bar{W} ;\left(x-a_{1}\right) \cdot p_{L}>0\right\}, \quad W_{2}=\left\{x \in \bar{W} ;\left(x-a_{1}\right) \cdot p_{L}<0\right\} .
$$


Then we have $\bar{W}_{1} \cup \bar{W}_{2}=\bar{W}, \bar{W}_{1} \cap \bar{W}_{2}=L \cap \bar{W}$. If $\arg \left(x-a_{1}\right)-\arg \left(a_{2}-a_{1}\right) \in(0, \pi)$

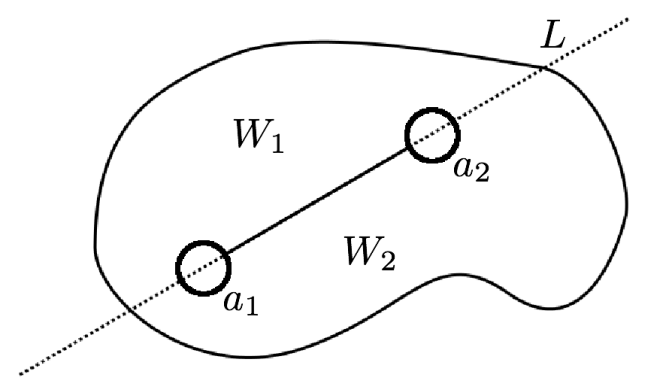

in $W_{1}$, then we also have $\arg \left(x-a_{2}\right)-\arg \left(a_{2}-a_{1}\right) \in(0, \pi)$, which implies

$$
\arg \left(x-a_{1}\right)-\arg \left(x-a_{2}\right) \not \equiv \pi \bmod 2 \pi \mathbb{Z} \text { on } W_{1} .
$$

The above is also obtained similarly when $\arg \left(x-a_{1}\right)-\arg \left(a_{2}-a_{1}\right) \in(-\pi, 0)$ in $W_{1}$ or in $W_{2}$. Moreover, we find

$$
\arg \left(x-a_{1}\right)-\arg \left(x-a_{2}\right) \equiv\left\{\begin{array}{ll}
0 & \text { on } L \cap \bar{W} \cap \Gamma^{c}, \\
\pi & \text { on } \Gamma
\end{array}\right\} \quad \bmod 2 \pi \mathbb{Z} .
$$

Thus $u \equiv \pi$ gives the above $\Gamma$ by $(2.8)$.

Additive construction. We give here an additive method of constructing $u$ satisfying

$$
\Gamma_{1} \cup \Gamma_{2}=\{x \in \bar{W} ; u(x)-\theta(x) \equiv 0 \quad \bmod 2 \pi \mathbb{Z}\}
$$

from the initial data $u_{1}$ and $u_{2}$ describing $\Gamma_{1}$ and $\Gamma_{2}$ as

$$
\Gamma_{i}=\left\{x \in \bar{W} ; u_{i}(x)-\theta_{i}(x) \equiv 0 \quad \bmod 2 \pi \mathbb{Z}\right\} \quad \text { for } i=1,2,
$$

respectively. Note that in this case $\theta \equiv \theta_{1}+\theta_{2} \bmod 2 \pi \mathbb{Z}$.

First, we prepare a modification of continuous function which still describes the same spirals. Let $H_{1}: \mathbb{R} \rightarrow \mathbb{R}$ be a function defined as

$$
H_{1}(\sigma)= \begin{cases}-1 & \text { if } \sigma \leq-1 \\ \sigma & \text { if }-1<\sigma<1 \\ +1 & \text { if } \sigma \geq 1\end{cases}
$$

Then, the functions

$$
v_{i}(x):=\Theta_{i}(x)+2 \pi k_{i}(x)+\pi H_{1}\left(\lambda_{i}\left[u_{i}(x)-\left(\Theta_{i}(x)+2 \pi k_{i}(x)\right)\right]\right)
$$

are still continuous and satisfy

$$
\Gamma_{i}=\left\{x \in \bar{W} ; v_{i}(x)-\theta_{i}(x) \equiv 0 \quad \bmod 2 \pi \mathbb{Z}\right\} \quad \text { for } i=1,2
$$

for any positive constants $\lambda_{i} \in[1 / \pi, \infty)$ and $i=1,2$, where $\Theta_{i}$ is a smooth branch of $\theta_{i}$ whose branch-cut line is on $\bigcup_{j=1}^{N}\left\{a_{j}+\left(x^{1}, 0\right) ; x^{1} \geq 0\right\}$, and $k_{i}: \bar{W} \rightarrow \mathbb{Z}$ are functions satisfying

$$
\left|u_{i}(x)-\left(\Theta_{i}(x)+2 \pi k_{i}(x)\right)\right| \leq \pi \quad \text { for } x \in \bar{W}, i=1,2 .
$$


Thus, choose $\lambda_{i}>0$ so that

$$
\begin{aligned}
& \left\{x \in \bar{W} ;\left|v_{1}(x)-\left(\Theta_{1}(x)+2 \pi k_{1}(x)\right)\right|<\pi\right\} \\
& \quad \cap\left\{x \in \bar{W} ;\left|v_{2}(x)-\left(\Theta_{2}(x)+2 \pi k_{2}(x)\right)\right|<\pi\right\}=\emptyset
\end{aligned}
$$

and then $u(x)=v_{1}(x)+v_{2}(x)+\pi$ is continuous and satisfies (2.9).

Remark 2.7. Constructing a $u \in C(\bar{W})$ for a general simple spiral curve $\Gamma=\{P(s) ; s \in$ $[0, \ell]\}$ is more complicated. Here we shall propose one way to construct $u$ for general $\Gamma$ associated with the origin. Let $\hat{r}(s)$ and $\hat{\xi}_{k}(s)$ be satisfy

$$
P(s)=\hat{r}(s)\left(\cos \hat{\xi}_{k}(s), \sin \hat{\xi}_{k}(s)\right) \text { for } s \in[0, \ell],
$$

and $\hat{\xi}_{k}(0) \in[2 \pi k, 2 \pi(k+1))$ for $k \in \mathbb{Z}$. We consider domains $E_{k}$ enclosed by $\widehat{\Gamma}_{k} \cup \mathcal{C}_{1, k} \cup$ $\widehat{\Gamma}_{k+1} \cup \mathcal{C}_{2, k}$, where

$$
\widehat{\Gamma}_{k}:=\left\{\left(\hat{r}(s), \hat{\xi}_{k}(s)\right) ; s \in[0, \ell]\right\}
$$

and

$$
\begin{aligned}
& \mathcal{C}_{1, k}=\left\{(\rho, \xi) ; \xi \in\left[\hat{\xi}_{k}(0), \hat{\xi}_{k+1}(0)\right]\right\}, \\
& \mathcal{C}_{2, k}=\left\{(R, \xi) ; \xi \in\left[\hat{\xi}_{k}(\ell), \hat{\xi}_{k+1}(\ell)\right]\right\} .
\end{aligned}
$$

Let $\varphi \in C^{2}(E) \cap C([\rho, R] \times \mathbb{R})$ satisfy

$$
\left\{\begin{aligned}
\Delta_{r, \xi} \varphi(r, \xi) & =0 & & \text { for }(r, \xi) \in E, \\
\varphi(\rho, \xi) & =\hat{\xi}_{0}(0) & & \text { for }(\rho, \xi) \in \mathcal{C}_{1}, \\
\varphi(R, \xi) & =\hat{\xi}_{0}(\ell) & & \text { for }(R, \xi) \in \mathcal{C}_{2}, \\
\varphi\left(\hat{r}(s), \hat{\xi}_{k}(s)\right) & =\hat{\xi}_{0}(s) & & \text { for }\left(\hat{r}(s), \hat{\xi}_{k}(s)\right) \in \widehat{\Gamma},
\end{aligned}\right.
$$

where $E=\bigcup_{k \in \mathbb{Z}} E_{k}, \mathcal{C}_{i}=\bigcup_{k \in \mathbb{Z}} \mathcal{C}_{i, k}$ for $i=1,2, \widehat{\Gamma}=\bigcup_{k \in \mathbb{Z}} \widehat{\Gamma}_{k}$, and $\Delta_{r, \xi}=\partial^{2} / \partial r^{2}+\partial^{2} / \partial \xi^{2}$. Then, $u(x)=\varphi(|x|, \operatorname{Arg}(x))$, where $\operatorname{Arg}(x) \in[0,2 \pi)$ is the principal value of $\arg (x)$, is a function satisfying (2.8). In fact, $\psi(r, \xi):=\varphi(r, \xi)-\xi$ still satisfies

$$
\Delta_{r, \xi} \psi=0 \quad \text { in } E_{k}
$$

and thus $\psi$ attains its maximum or minimum on $\partial E_{k}=\widehat{\Gamma}_{k} \cup \widehat{\Gamma}_{k+1} \cup \mathcal{C}_{1, k} \cup \mathcal{C}_{2, k}$ by the maximum principle [PW67]. Moreover, from the last case in (2.11) we have

$$
\psi(r, \xi)=-2 \pi k \quad \text { for }(r, \xi) \in \widehat{\Gamma}_{k},
$$

i.e., $\psi$ is not a constant. Then we have

$$
-2 \pi(k+1)<\psi<-2 \pi k \text { in } E_{k},
$$

and thus

$$
u(x)-\arg (x) \equiv \varphi(|x|, \operatorname{Arg}(x))-\operatorname{Arg}(x)=\psi(|x|, \operatorname{Arg}(x)) \equiv 0 \quad \bmod 2 \pi \mathbb{Z}
$$

only on $\Gamma$. 
2.5. Evaluating the height function. It is of great interest to predict the growth rate of the crystal surface. Burton, Cabrera and Frank in [BCF51] calculate the growth rate of the surface with a single center by calculating the angle velocity of the rotating spiral. In this paper, we consider a general case that involve multiple centers. We construct a surface height function $h(t, x)$ from $\Gamma_{t}$ and obtain the mean growth rate $R_{h}\left(t ; t_{0}\right)$ of the surface in $\left[t_{0}, t\right]$ as

$$
R_{h}\left(t ; t_{0}\right)=\frac{1}{|W|} \int_{\bar{W}} \frac{h(t, x)-h\left(t_{0}, x\right)}{t-t_{0}} d x
$$

and the growth rate $R(t)$ as

$$
R(t)=R_{h}^{\prime}\left(t ; t_{0}\right)=\frac{1}{|W|} \int_{\bar{W}} h_{t}(t, x) d x .
$$

We construct $h(t, x)$ from the approximation by the theory of dislocations as in [HL68]. Here we assume that the vertical displacement of the surface by screw dislocations is small enough, and there is no horizontal displacement. Then, from the linear elasticity theory $h$ satisfies

$$
\Delta h=-h_{0} \operatorname{div} \delta_{\Gamma_{t}} \mathbf{n}
$$

where $h_{0}$ is a unit height of steps, and $\delta_{\Gamma_{t}}$ is the delta measure concentrated on $\Gamma_{t}$. Instead of solving (2.12) with a Neumann boundary condition as in [Sme00], we solve it analytically and derive an explicit formula for $h$. Let $\theta_{\Gamma_{t}}$ be the branch of $\theta$ given by (2.3) whose discontinuity is only on $\Gamma_{t}$. By a direct calculation we observe that

$$
h(t, x)=\frac{h_{0}}{2 \pi} \theta_{\Gamma_{t}}
$$

is a solution to (2.12). Since the jump of $\theta_{\Gamma_{t}}$ is $-2 \pi$ in the direction of the normal, the multiplier $1 / 2 \pi$ in front of $\theta_{\Gamma_{t}}$ is necessary so that (2.13) solves $(2.12)$. Hence, $h(t, x)$ can be evaluated conveniently from the solution $u$ of $(2.4)-(2.5)$ as described in the following.

Let $k(t, x) \in \mathbb{Z}$ be such that

$$
|u(t, x)-(\Theta(x)+2 \pi k(t, x))| \leq \pi
$$

where $\Theta(x)=\sum_{j=1}^{N} m_{j} \Theta_{j}(x)$ and $\Theta_{j}: \bar{W} \rightarrow[0,2 \pi)$ is a principal value of $\arg \left(x-a_{j}\right)$. Let $H: \mathbb{R} \rightarrow \mathbb{R}$ be the Heaviside function, i.e.,

$$
H(\sigma)= \begin{cases}-1 & \text { if } \sigma<0 \\ +1 & \text { if } \sigma \geq 0\end{cases}
$$

We now define

$$
h(t, x)=\frac{h_{0}}{2 \pi}[\Theta(x)+2 \pi k(t, x)+\pi H(u(t, x)-(\Theta(x)+2 \pi k(t, x)))] .
$$

This is our desired function to describe the height of the crystal surface. 


\section{Numerical Simulations}

In this section we present a few results of numerical experiments.

We set the domain $\Omega=[-1,1]^{2}$, uniform grid spacing $\delta=10^{-2}$, and time step $\tau=\delta^{2} / 4$ for numerical simulations in this section. The lattice points are denoted by $\left(t^{k}, x_{i, j}\right)=$ $(\tau k, \delta i, \delta j)$ for $-100 \leq i, j \leq 100$. In this section we use the equation

$$
V=v_{\infty}\left(1-\rho_{c} \kappa\right)
$$

instead of (1.1) for consistency with [BCF51]. The corresponding level set equation is given in

$$
u_{t}-v_{\infty}|\nabla(u-\theta)|\left\{\rho_{c} \operatorname{div} \frac{\nabla(u-\theta)}{|\nabla(u-\theta)|}+1\right\}=0 \quad \text { in }(0, T) \times W .
$$

Note that $v_{\infty}$ denotes the evolution speed of a straight line, and $\rho_{c}$ denotes the critical radius such that a disc shrinks if its radius is less than $\rho_{c}$. Solving (1.1) with $C=1 / \rho_{c}$, and rescaling $t$ to $v_{\infty} \rho_{c} t$, one obtains the dynamics of spirals prescribed in (3.1).

3.1. Discretization. In this paper we solve (2.4)-(2.5) with a typical explicit finite difference scheme; see e.g. [OF01],[TO05]. We shall give only a few remarks which are rather special to our problem.

One of the specific difficulties is in treating the sheet structure function $\theta$ when we apply finite differencing to the terms $u-\theta$ in (2.4) or (2.5). The function $\theta$ will be evaluated numerically in a neighborhood of each branch-cut line of $\arg \left(x-a_{j}\right)$ so that it is smooth there and we do not perform finite difference across the projected discontinuity of $\arg \left(x-a_{j}\right)$.

Writing $w=u-\theta$ formally, the equation (2.4) appears in the form

$$
\begin{aligned}
& u_{t}-v_{\infty} \mathrm{I}-v_{\infty} \rho_{c} \mathrm{II}=0, \\
& \mathrm{I}=|\nabla w|, \\
& \mathrm{II}=|\nabla w| \operatorname{div} \frac{\nabla w}{|\nabla w|} .
\end{aligned}
$$

More precisely, we denote

$$
\begin{aligned}
& \mathrm{I}=\sqrt{\left|\tilde{\partial}_{x} w\right|^{2}+\left|\tilde{\partial}_{y} w\right|^{2}}, \\
& \mathrm{II}=\sqrt{\left|\hat{\partial}_{x} w\right|^{2}+\left|\hat{\partial}_{y} w\right|^{2}} \operatorname{div} \frac{\nabla w}{|\bar{\nabla} w|}
\end{aligned}
$$


with $w_{i, j}^{k}=w\left(t^{k}, x_{i, j}\right)$ on a lattice $\left(t^{k}, x_{i, j}\right)$ with uniform grid spacing $\delta>0$ in the $x$ - and the $y$ - dimensions. If $v_{\infty}>0,\left|\tilde{\partial}_{x} w\right|$ and $\left|\hat{\partial}_{x} w\right|$ are discretized differently as follows:

$$
\begin{aligned}
\left|\tilde{\partial}_{x} w\right| & =\max \left\{\max \left\{\tilde{\partial}_{x}^{+} w, 0\right\},-\min \left\{\tilde{\partial}_{x}^{-} w, 0\right\}\right\}, \\
\left|\hat{\partial}_{x} w\right| & = \begin{cases}\max \left\{\left|\partial_{x}^{+} w\right|,\left|\partial_{x}^{-} w\right|\right\} & \text { if }\left|\partial_{x}^{\circ} w\right| \ll 1, \\
\left|\partial_{x}^{\circ} w\right| & \text { otherwise, }\end{cases} \\
\partial_{x}^{ \pm} w & =\frac{w_{i \pm 1, j}^{k}-w_{i, j}^{k}}{ \pm \delta}, \quad \partial_{x}^{\circ} w=\frac{w_{i+1, j}^{k}-w_{i-1, j}^{k}}{2 \delta}, \\
\tilde{\partial}_{x}^{ \pm} w & =\frac{w_{i \pm 1, j}^{k}-w_{i, j}^{k}}{ \pm \delta} \mp \frac{1}{2} \mu\left(\frac{w_{i \pm 2, j}^{k}-2 w_{i \pm 1, j}^{k}+w_{i, j}^{k}}{\delta^{2}}, \frac{w_{i+1, j}^{k}-2 w_{i, j}^{k}+w_{i-1, j}^{k}}{\delta^{2}}\right), \\
\mu(p, q) & = \begin{cases}p & \text { if }|p|<q, \\
q & \text { otherwise }\end{cases}
\end{aligned}
$$

If $v_{\infty}<0$, then $\tilde{\partial} w_{x}$ is discretized by

$$
\left|\tilde{\partial}_{x} w\right|=\max \left\{-\min \left\{\tilde{\partial}_{x}^{+} w, 0\right\}, \max \left\{\tilde{\partial}_{x}^{-} w, 0\right\}\right\} .
$$

The terms $\left|\tilde{\partial}_{y} w\right|,\left|\hat{\partial}_{y} w\right|$ are defined analogously as above.

The curvature term $\operatorname{div}(\nabla w /|\bar{\nabla} w|)$ is discretized as

$$
\begin{array}{r}
\operatorname{div} \frac{\nabla w}{|\bar{\nabla} w|}=\frac{1}{\delta}\left(\frac{\partial_{x}^{+} w}{\sqrt{\varepsilon^{2}+\left(\partial_{x}^{+} w\right)^{2}+\left(\bar{\partial}_{y}^{+} w\right)^{2}}}-\frac{\partial_{x}^{-} w}{\sqrt{\varepsilon^{2}+\left(\partial_{x}^{-} w\right)^{2}+\left(\bar{\partial}_{y}^{-} w\right)^{2}}}\right. \\
\left.+\frac{\partial_{y}^{+} w}{\sqrt{\varepsilon^{2}+\left(\bar{\partial}_{x}^{+} w\right)^{2}+\left(\partial_{y}^{+} w\right)^{2}}}-\frac{\partial_{y}^{-} w}{\sqrt{\varepsilon^{2}+\left(\bar{\partial}_{x}^{-} w\right)^{2}+\left(\partial_{y}^{-} w\right)^{2}}}\right)
\end{array}
$$

with a small parameter $\varepsilon>0$, where $\bar{\partial}_{x}^{ \pm} w$ is discretized as

$$
\bar{\partial}_{x}^{ \pm} w=\frac{\left(w_{i+1, j \pm 1}^{k}+w_{i+1, j}^{k}\right)-\left(w_{i-1, j \pm 1}^{k}+w_{i-1, j}^{k}\right)}{4 \delta} .
$$

The term $\bar{\partial}_{y}^{ \pm} w$ is also defined analogously as above.

We now discuss the treatment of Neumann boundary condition for the boundary of a small region $U$ that contains the spiral center. We consider two idealized situations. The first one being that $U$ corresponds to a disc centering at a grid node $x_{i, j}$ with a radius that is smaller than $\delta / 2$. The second situation corresponds to $U$ being a disc with a small radius which is independent of the grid spacing. In the first situation, we assign different fictitious values to $w_{i, j}$ depending on the finite difference stencil used in discretizing the PDE at a grid node nearby $x_{i, j}$, assuming that $x_{i, j}$ is in the computational domain. More precisely, if the PDE is discretized on $x_{i-1, j}$, then we assign the fictitious value of $w_{i, j}$ to be $w_{i-1, j}$. The other fictitious values of $w_{i, j}$ are assigned accordingly. We remark, however, that this approach results in relatively larger error in the front propagation speed near the spiral center.

In the second idealized situation, we further assume that the mesh size $\delta$ is smaller than the radius of $U$, and that explicit time stepping such as forward Euler or some explicit 
Runge-Kutta method is used to time discretization. In this setting, we may consider $\partial U$ as an implicit interface, and extend the values of $w$ outside of $U$ following the approach which is called "velocity extension" in the level set method literature; see e.g. [OF01], or more specifically [CT08].

3.2. Single center with multiple spirals. One of advantages over the Smereka's formulation is that it is easy to treat the situation there is one center with multiple spirals. This situation is described by

$$
\Gamma_{t}:=\{x \in \bar{W} ; u(t, x)-m \theta(x) \equiv 0 \quad \bmod 2 \pi \mathbb{Z}\}
$$

with $\theta(x)=\arg x$ and $m \in \mathbb{Z} \backslash\{0\}$. Here we have assumed that the center is the origin. The dynamics is given by

$$
\begin{aligned}
u_{t}-v_{\infty}|\nabla(u-m \theta)|\left\{\rho_{c} \operatorname{div} \frac{\nabla(u-m \theta)}{|\nabla(u-m \theta)|}+1\right\}=0 & \text { in } \quad(0, T) \times W, \\
\langle\vec{\nu}, \nabla(u-m \theta)\rangle & =0 \quad \text { on } \quad(0, T) \times \partial W .
\end{aligned}
$$

Then we find evolving $|m|$ spirals as $\Gamma_{t}=\bigcup_{k=0}^{|m|-1} \Gamma_{k, t}$ and

$$
\Gamma_{k, t}=\{x \in \bar{W} ; u(t, x)-m \theta(x) \equiv 2 \pi k \quad \bmod 2 \pi|m| \mathbb{Z}\} .
$$

To describe this situation by Smereka's formulation we need $2|m|$ auxiliary function and thus system of $2|m|$ equations.

Figure 3.1 are the evolution of triple spirals associated with the origin by

$$
\left.V=5(1-0.03 \kappa) \quad \text { (i.e., } v_{\infty}=5, \rho_{c}=0.03\right) .
$$

The initial curve is chosen as

$$
\Gamma_{0}=\bigcup_{i=1}^{3}\left\{r\left(\cos \frac{2(i-1)}{3} \pi, \sin \frac{2(i-1)}{3} \pi\right) ; r>0\right\} .
$$

In this case we choose $u_{0}(x) \equiv 0$.

3.3. Co-rotating spirals. Consider the case of $N$ screw dislocations with the same rotational orientations. We say such a case simply co-rotating spirals. To describe this situation we consider (2.4)-(2.5) with

$$
\theta(x)=m \sum_{i=1}^{N} m_{i} \arg \left(x-a_{i}\right)
$$

where $m_{i} \in \mathbb{N}$ is the number of spirals associated with $a_{i}$, and $m \in\{ \pm 1\}$ is the constant chosen by the rotational orientations, i.e., $m=1$ if the orientations all spirals are counterclockwise, and $m=-1$ if those are clockwise.

Note that there are no connecting spirals for co-rotating case. Then, if $\Gamma_{0}$ is the union of lines, $\Gamma_{0}$ is given as

$$
\begin{aligned}
\Gamma_{0} & =\bigcup_{i=1}^{N} \bigcup_{j=1}^{m_{i}} L_{i, j}, \\
L_{i, j} & =\left\{a_{i}+r\left(\cos \alpha_{i, j}, \sin \alpha_{i, j}\right) \in \bar{W} ; r>0\right\},
\end{aligned}
$$

where $\alpha_{i, j} \in \mathbb{R}$ is a constant. 

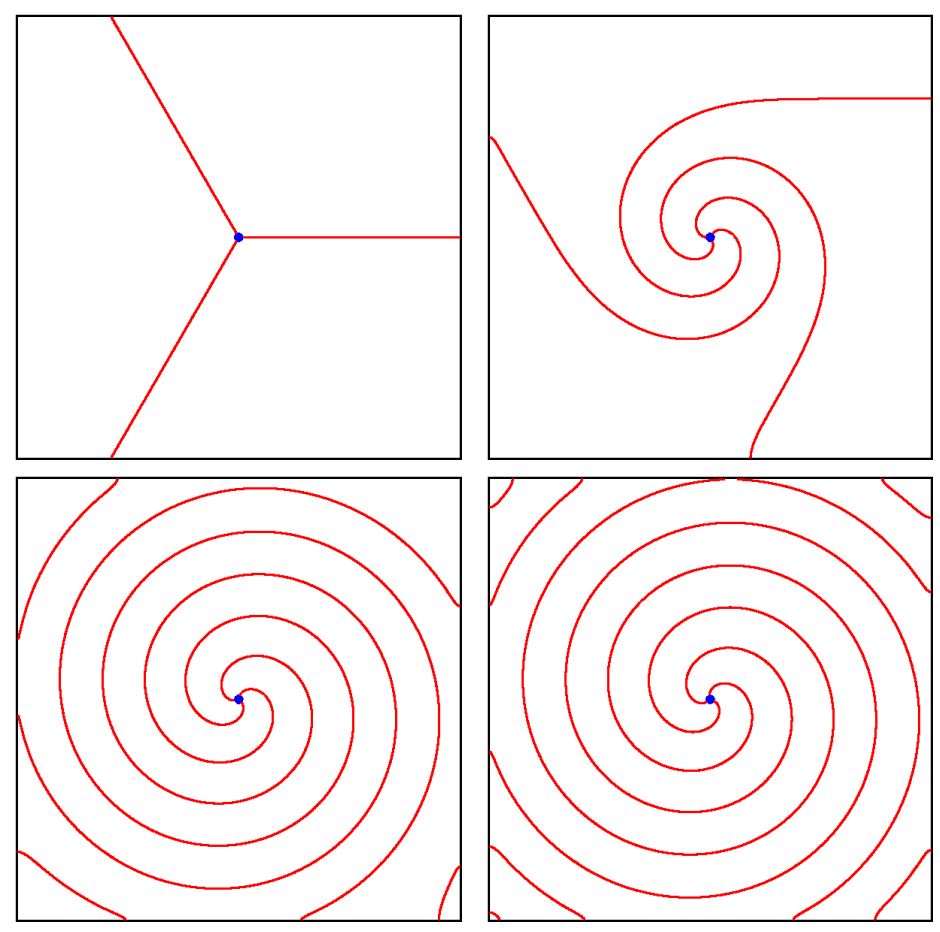

FigURE 3.1. Motion of triple spirals associated with the origin by $V=$ $5(1-0.03 \kappa)$. Each profile is at $t=0,0.1250,0.250$ and $t=0.50$ from left top to right bottom.

A simplest but nontrivial situation is the case of $N=2, m_{1}=m_{2}=1$ and $\Gamma_{0}=$ $L_{1,1} \cup L_{2,1}$ is given by

$$
L_{1,1}=\left\{a_{1}+r\left(a_{1}-a_{2}\right) \in \bar{W} ; r>0\right\}, \quad L_{2,1}=\left\{a_{2}+r\left(a_{2}-a_{1}\right) \in \bar{W} ; r>0\right\}
$$

with counter-clockwise orientation. In this case $\theta$ and $u_{0}$ are of the form

$$
\begin{aligned}
& \theta(x)=\arg \left(x-a_{1}\right)+\arg \left(x-a_{2}\right), \\
& u_{0}(x) \equiv 0 .
\end{aligned}
$$

Note that we set $\theta(x)=-\arg \left(x-a_{1}\right)-\arg \left(x-a_{2}\right)$ instead of the above if the rotational orientations of the curve are clockwise. The figure 3.2 is the simulation with

$$
\left\{\begin{array}{l}
a_{1}=(-0.35,0), a_{2}=(0.35,0) \\
V=5(1-0.02 \kappa) \quad\left(v_{\infty}=5, \rho_{c}=0.02\right) .
\end{array}\right.
$$

We also obtain the surface height function from a solution of the level set equation with the method in $\S 2.5$ (See figure 3.3.).

One of advantages of our method is that our method enables us to set different numbers of spirals for several centers, i.e., describing the situations for more general cases of $m$ and $m_{i}$. Such situation seems to be impossible to treat by Smereka's approach. We now assume that an initial curve is given by (3.2) and (3.3) with counter-clockwise orientation. Then, from the additive construction we first choose $u_{i, j} \in C(\bar{W})$ satisfying

$$
L_{i, j}=\left\{x \in \bar{W} ; u_{i, j}(x)-\arg \left(x-a_{i}\right)=0 \quad \bmod 2 \pi \mathbb{Z}\right\},
$$



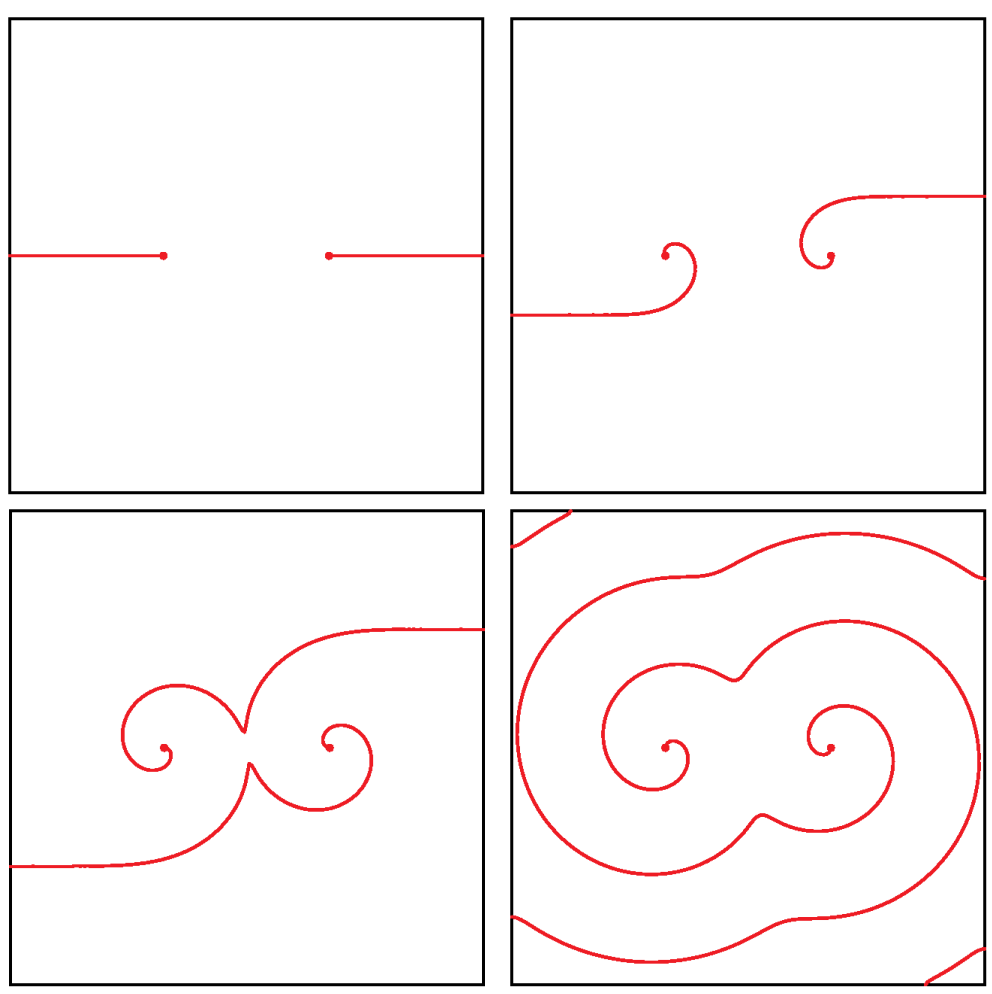

Figure 3.2. The simulation of co-rotating spirals by (3.5) and (3.4) at time $t=0, t=0.05, t=0.1, t=0.5$ from left-top to right-bottom.

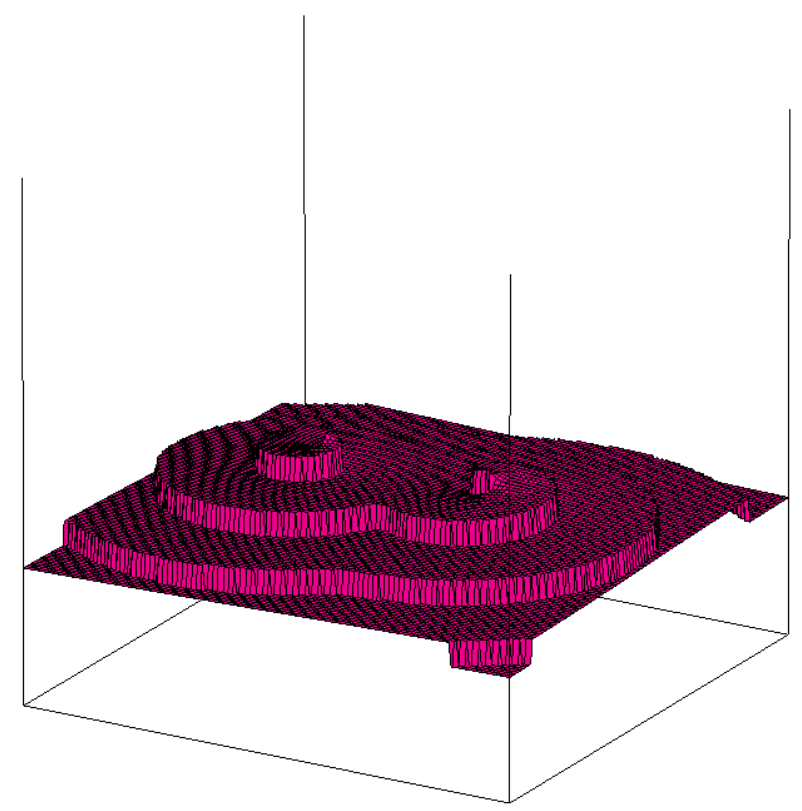

Figure 3.3. The profile of the surface at $t=0.5$ from figure 3.2 , which is reconstructed from the numerical solution of the level set equation. 
and one observe that $u_{i, j}(x) \equiv \alpha_{i, j}$ from the initialization of a line step in $\S 2.4$. Next, we modify $u_{i, j}$ as

$$
v_{i, j}(x)=\Theta_{i}(x)+2 \pi k_{i, j}(x)+\pi H_{1}\left(\lambda_{i, j}\left\{u_{i, j}(x)-\left(\Theta_{i}(x)+2 \pi k_{i, j}(x)\right)\right\}\right),
$$

with constants $\lambda_{i, j}>1 / \pi$, where $H_{1}$ is a function defined as $(2.10), \Theta_{i}: \bar{W} \rightarrow[0,2 \pi)$ is a principal value of $\arg \left(x-a_{i}\right)$, and $k_{i, j}: \bar{W} \rightarrow \mathbb{Z}$ is a function satisfying

$$
-\pi \leq u_{i, j}(x)-\left(\Theta_{i}(x)+2 \pi k_{i, j}(x)\right)<\pi \quad \text { for } x \in \bar{W} .
$$

Here we choose $\lambda_{i, j}$ as

$$
\Lambda_{i_{1}, j_{1}} \cap \Lambda_{i_{2}, j_{2}}=\emptyset \quad \text { whenever }\left(i_{1}, j_{1}\right) \neq\left(i_{2}, j_{2}\right),
$$

where

$$
\Lambda_{i, j}=\left\{x \in \bar{W} ;\left|v_{i, j}(x)-\left(\Theta_{i}(x)+2 \pi k_{i, j}(x)\right)\right|<\pi\right\}
$$

Then, we set

$$
u_{0}(x)=\sum_{i=1}^{N} \sum_{j=1}^{m_{i}}\left(v_{i, j}(x)+\pi\right)-\pi .
$$

Note that $v_{i, j} \in C(\bar{W})$ and satisfies

$$
L_{i, j}=\left\{x \in \bar{W} ; v_{i, j}(x)-\arg \left(x-a_{i}\right) \equiv 0 \quad \bmod 2 \pi \mathbb{Z}\right\}
$$

if $\lambda_{i, j}>1 / \pi$. The condition (3.8) is a sufficient condition to give an initial curve by $u_{0}$. Here is an example of simulation of general situation in figure 3.4 with (3.5) and

$$
\begin{aligned}
\theta(x) & =\arg \left(x-a_{1}\right)+2 \arg \left(x-a_{2}\right), \\
L_{1,1} & =\left\{a_{1}+r(\cos \pi, \sin \pi) ; r>0\right\}, \\
L_{2,1} & =\left\{a_{2}+r(\cos (-\pi / 3), \sin (-\pi / 3)) ; r>0\right\}, \\
L_{2,2} & =\left\{a_{2}+r(\cos (\pi / 3), \sin (\pi / 3)) ; r>0\right\} .
\end{aligned}
$$

To give an initial datum $u_{0}$ for $\Gamma_{0}=\bigcup_{i=1}^{2} \bigcup_{j=1}^{m_{i}} L_{i, j}$ we set

$$
\alpha_{1,1}=\pi, \alpha_{2,1}=-\frac{\pi}{3}, \alpha_{2,2}=\frac{\pi}{3}, \quad \lambda_{1,1}=\lambda_{2,1}=\lambda_{2,2}=\frac{3}{\pi}
$$

Remark 3.1. When the curves have clockwise orientations, we set $\tilde{u}_{i, j}=-u_{i, j}$ to obtain $L_{i, j}=\left\{x \in \bar{W} ; \tilde{u}_{i, j}(x)-\theta_{i}^{-}(x) \equiv 0 \bmod 2 \pi \mathbb{Z}\right\}$ with $\theta_{i}^{-}=-\arg \left(x-a_{i}\right)$ and set $\tilde{u}_{i, j}$ and the principal value $\Theta_{i}^{-}(x)$ of $\theta_{i}^{-}(x)$ instead of $u_{i, j}$ and $\Theta_{i}$ in (3.6)-(3.9) to obtain $u_{0}$ as (3.10).

One advantage of our method over the Smereka's method [Sme00] is that our method is able to verify activity of group of screw dislocations and compare it with a single screw dislocation with multiple steps.

Burton, Cabrera and Frank [BCF51, 9] pointed out that the activity of co-rotating spirals depends on the distance of the centers. They first consider the case of a pair of corotating spirals, and pointed out that the activity of a co-rotating pair is indistinguishable from that of one screw dislocation if the centers are far apart, and, however, should be 

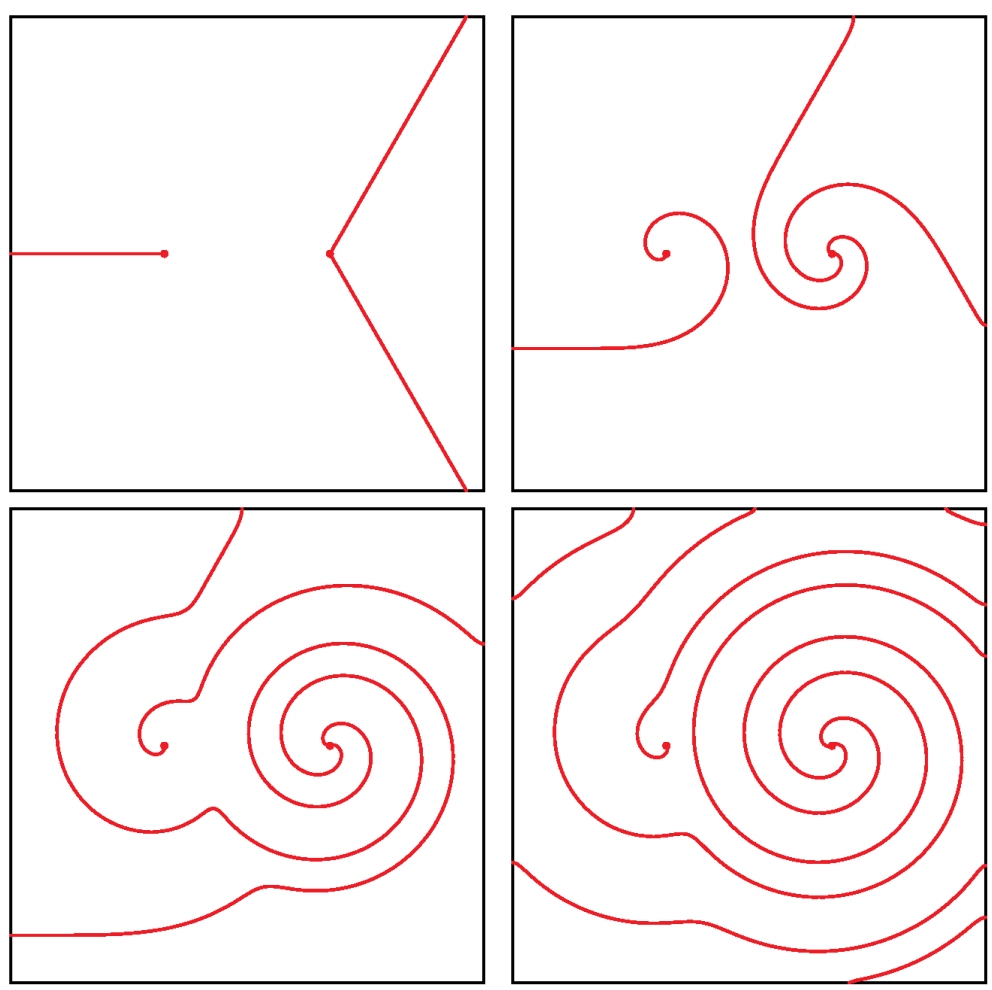

FiguRE 3.4. Co rotating spirals with different numbers of spiral steps for each screw dislocations at $t=0$ on left top, $t=0.08, t=0.16, t=0.24$ on right bottom.

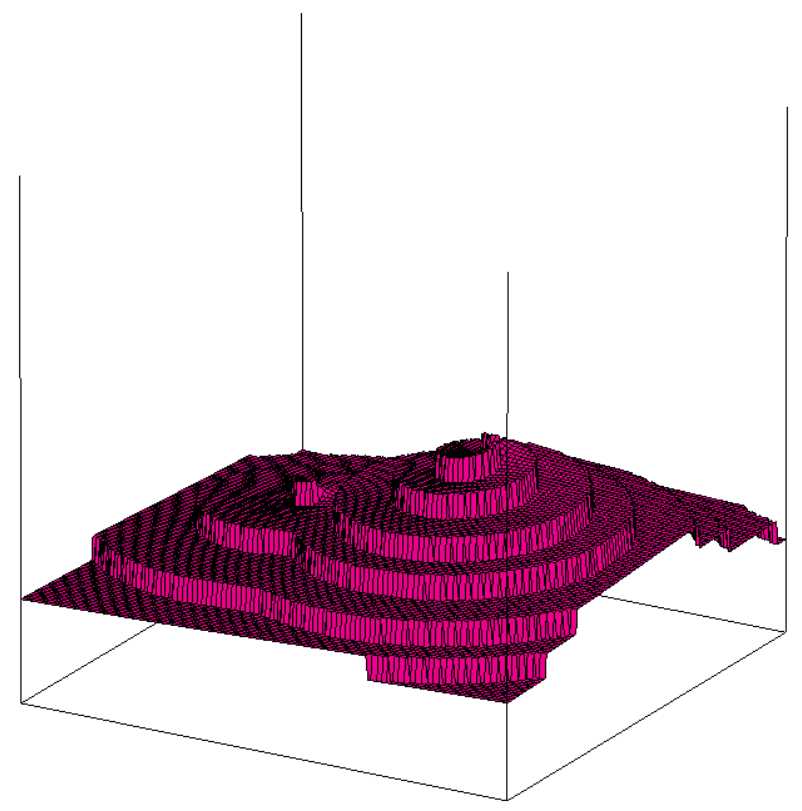

FigURE 3.5. Reconstructed surface at $t=0.24$ from the numerical solution in 3.4 . 
twice of one screw dislocation if the distance of the centers is much less than $\rho_{c}$, i.e., $\left|a_{1}-a_{2}\right| \ll \rho_{c}$ for the pair of centers $a_{1}$ and $a_{2}$. They also pointed out that the profile of corotating spirals would be effectively two symmetric branches of the complete Archimedean spirals, $r=2 \rho_{c} \theta$ and $r=2 \rho_{c}(\theta+\pi)$ in the limiting case as $\left|a_{1}-a_{2}\right| \rightarrow 0$ where $r, \theta$ are the variables in the polar coordinates. By considering the spirals associated with $a_{1}$ and $a_{2}$ defined by the Archimedeans $r=2 \rho_{c} \theta$ and $2 \rho_{c}(\theta+\pi)$, they observed that the spirals did not collide with each other if and only if $\left|a_{1}-a_{2}\right|<2 \pi \rho_{c}$ (see Figure 3.6). Thus they discuss the activities and the profiles of spirals according to the centers being "close" $\left(\left|a_{1}-a_{2}\right|<2 \pi \rho_{c}\right)$ or "far apart" $\left(\left|a_{1}-a_{2}\right| \geq 2 \pi \rho_{c}\right)$.

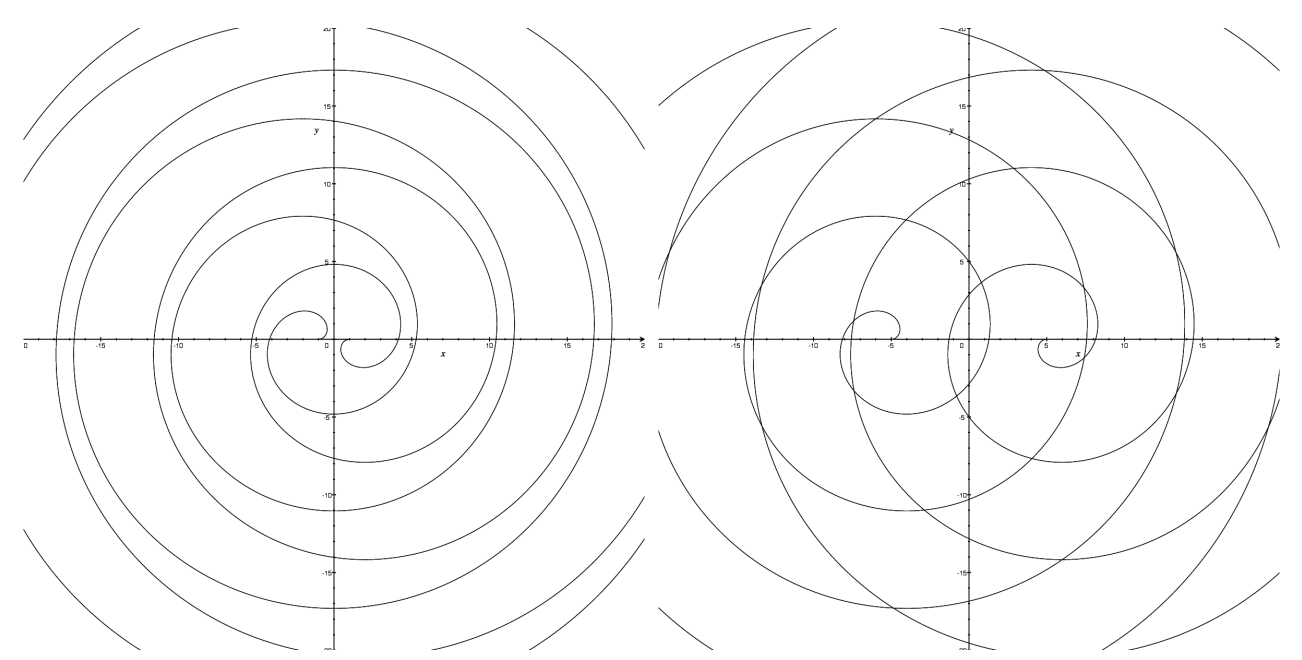

Figure 3.6. Two archimedeans: $r=2 \rho_{c} \theta$ centering at $a_{1}=(-\alpha, 0)$ and its half turn $r=2 \rho_{c}(\theta+\pi)$ centering at $a_{2}=(\alpha, 0)$. In these figures $\rho_{c}=1 / 2$, $\alpha=1$ in the left figure, and $\alpha=5$ in the right figure, respectively.

Here we present a few simulations that verify the two cases discussed in [BCF51]. In Figure 3.7 we show three simulations involving respectively two spirals connecting to a single center at $(0,0)$, two spirals each connecting to one of the two centers at $( \pm 0.02,0)$, and to $( \pm 0.2,0)$. The evolution equation is

$$
V=5(1-0.02 \kappa)
$$

i.e., $\rho_{c}=0.02$. We choose

$$
u_{0}(x)=0
$$

for all the case. Figure 3.2 shows a simulation for the farthest case with the same equation as the simulations in figure 3.7 .

The simulation presented in the middle column corresponds to the case $\left|a_{1}-a_{2}\right|=$ $0.04<2 \pi \rho_{c}$. In the setup of the simulation we take $a_{1}$ and $a_{2}$ as close as possible, so that there are three grid points between the pair to see the performance of the proposed method for spirals with centers that are closely positioned on the grid level. Even though we cannot say that this pair falls into the regime $\left|a_{1}-a_{2}\right| \ll \rho_{c}$, our simulations show that the corresponding profile is significantly different from the case in which the pair consists of centers at $( \pm 0.2,0)$. A crucial difference is that the curve includes some concave points. 


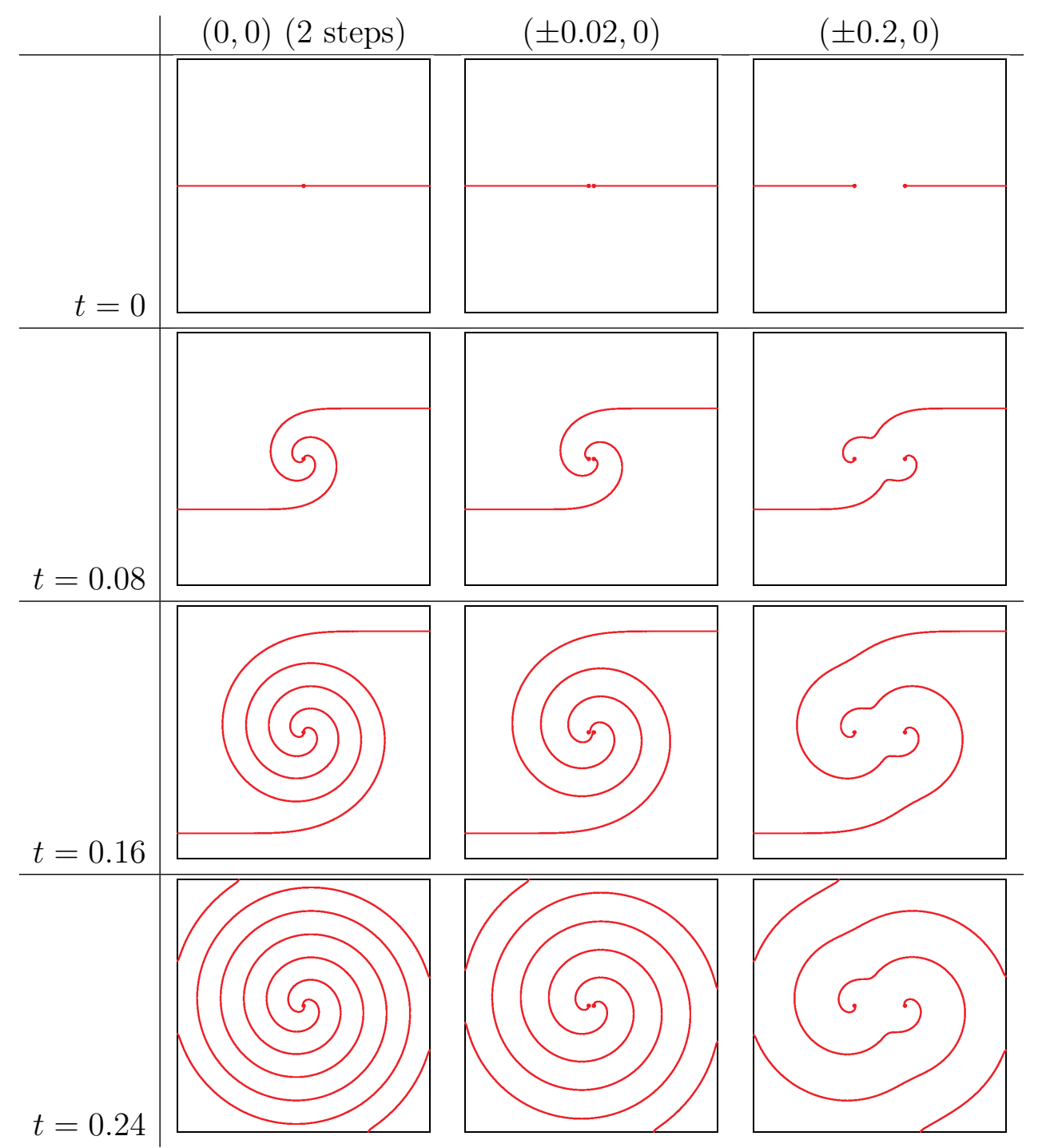

Figure 3.7. Comparison of co-rotating spirals by distances of pairs, and single center with two branches. The pictures are a single center with two branches, at $( \pm 0.02,0)$ and $( \pm 0.2,0)$ from left to right, and $t=0,0.08,0.16$ and 0.24 from top to bottom.

One observes that the line tracking points where the curve is concave almost agrees with the locus of intersections and forms an S-shape.

Remark 3.2. In [OTG] we shall discuss the growth rate of the surface for the case of centers $( \pm 0.02,0)$ is very close to the case of a single center with double spirals, and the case $( \pm 0.2,0)$ is caught up by a single spiral case.

Burton et al [BCF51] observed by a heuristic argument that a set of centers on one line plays a role of a single center with multiple activity when the distances between two neighboring centers one the line is less than $2 \pi \rho_{c}$. Such a set is called a group (or system) 
of centers (or co-rotating spirals). They also presented formulae predicting the activities between a group of co-rotating spirals and a single one (see Remark 3.3).

We verify the difference of profiles between some cases of systems by $N(\geq 2)$ co-rotating spirals. Figure 3.8 is a results of simulations on 4 centers $( \pm a, 0),( \pm b, 0)(a>b>0)$ with counter-clockwise orientations. The evolution equation is (3.11). The first examination is with $a=0.06$ and $b=0.02$, the second one is $a=0.15, b=0.11$. According to [BCF51,9]

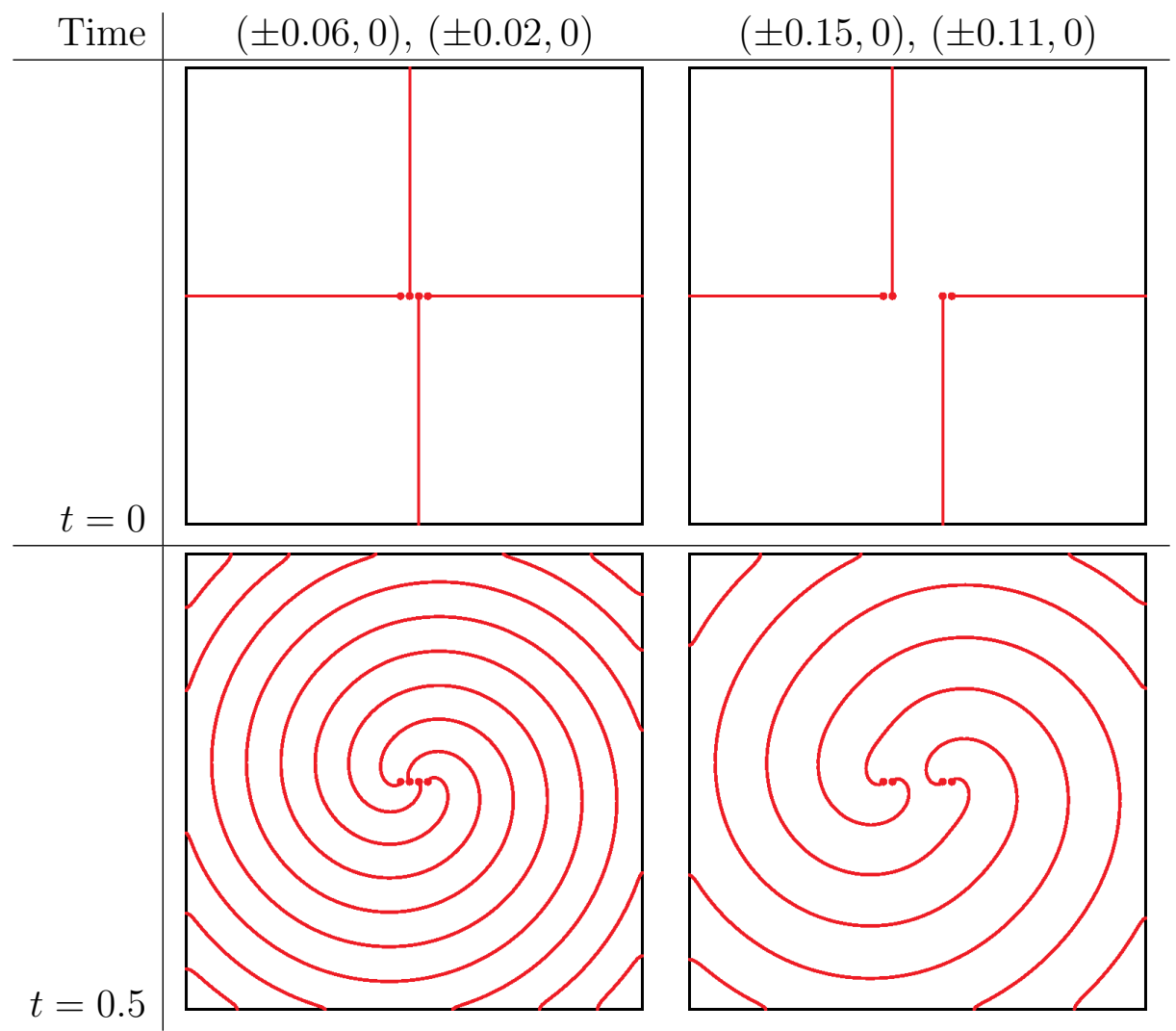

Figure 3.8. Comparison of profiles of spirals at time $t=0$ (top) and $t=$ 0.5 (bottom) by 4 centers with the same rotational orientations. The left one is with $( \pm 0.06),(0.02,0)$, and the right one is $( \pm 0.15,0.11)$.

the first one should be regarded as one group of four centers, and the second one should be two pairs.

In these tests we define the initial curve $\Gamma_{0}=L_{1} \cup L_{2} \cup L_{3} \cup L_{4}$ to be

$$
\begin{array}{ll}
L_{1}=\left\{a_{1}+(-r, 0) ; r>0\right\}, & a_{1}=(-a, 0), \\
L_{2}=\left\{a_{2}+(0,-r) ; r>0\right\}, & a_{2}=(-b, 0), \\
L_{3}=\left\{a_{3}+(0, r) ; r>0\right\}, & a_{3}=(b, 0), \\
L_{4}=\left\{a_{4}+(r, 0) ; r>0\right\}, & a_{4}=(a, 0) .
\end{array}
$$

Here we have used the simple notations $L_{i}$ instead of $L_{i, 1}$ since each centers have single line. Here and hereafter we will use similar notations $\alpha_{i}, \lambda_{i}$ instead of $\alpha_{i, 1}, \lambda_{i, 1}$ if $\Gamma_{0}$ is given by (3.2) and (3.3), and each the center is connected to a single line. In these 
numerical experiments we choose $\theta=\sum_{i=1}^{4} \arg \left(x-a_{i}\right)$, and

$$
\alpha_{1}=\pi, \alpha_{2}=-\pi / 2, \alpha_{3}=\pi / 2, \alpha_{4}=0, \quad \text { and } \lambda_{i}=4 / \pi \quad \text { for } i=1,2,3,4
$$

to construct $u_{0}$ as (3.10).

Remark 3.3. (i) The growth height and rate in $\S 2.5$ enables us to find the essential difference between the case $( \pm 0.06,0),( \pm 0.02,0)$ and $( \pm 0.15,0),( \pm 0.11,0)$. According to [BCF51, 9], the resultant activity of a group of $N$ co-rotating spirals is $N /\left(1+l\left(2 \pi \rho_{c}\right)^{-1}\right)$ times that of a single spiral if the group is on a line whose length is $l$. The growth rate obtained by our examination implies that the numerical growth rate by co-rotating screw dislocations at $( \pm 0.15,0),( \pm 0.11,0)$ is closer to the case of two pairs of dislocations with line length 0.04 than that by the group of 4 screw dislocations with line length 0.30 . We shall discuss this subject in one of our forthcoming paper [OTG].

(ii) There is no explicit definition of activity of a group of screw dislocations in [BCF51]. A reasonable definition of the activity of a group is the growth rate of the surface around the group.

(iii) There is a quantity which is called the "strength" of a group in [BCF51]. The strength should be defined as the sum of the all signed numbers of spirals associated with centers joined in the group.

We conclude this section by examining a more general group of co-rotating spirals, for which Burton et al [BCF51] discussed heuristically. Here it is convenient to introduce a notion of $\delta$-arcwise connecticity. We say that a discrete set $\mathcal{A}$ in $\mathbb{R}^{2}$ is $\delta$-arcwise connected if any two points $a_{1}, a_{2} \in \mathcal{A}$ is connected by a $\delta$-chain. Here a $\delta$-chain is a zig-zag line whose vertices agree with some points in $\mathcal{A}$ and the length of each segment is less than $\delta$. As we mentioned above, if the distance of a co-rotating pair is less than $2 \pi \rho_{c}$, then the pair is effectively a single center which has twice activity. Moreover, if a third center in the domain is also less than $2 \pi \rho_{c}$ distance to the closest center in the pair, then these three centers are also regarded as a single center with about triple activity. Consequently, a set of $2 \pi \rho_{c}$-arcwise connected centers generate a group of centers, i.e., plays a role of a single center with multiple strength. For example, centers in the left figures of Figure 3.8 generate a group of 4 centers, and those in the right figures generate two pairs (not a group of 4 centers).

In general, however, the group of centers may develop a pit in the surface of the crystal. We consider a group of centers which are at $a_{1}=(0.16,0), a_{2}=(0.08,0.15), a_{3}=$ $(-0.08,0.15), a_{4}=(-0.16,0), a_{5}=(-0.08,-0.15)$, and $a_{6}=(0.08,-0.15)$. Set the initial line as

$$
L_{i}=\left\{a_{i}+r(\cos \pi(i-1) / 3, \sin \pi(i-1) / 3) ; r>0\right\} \quad \text { for } i=1,2,3,4,5,6,
$$

and evolve $\Gamma_{0}=\cup_{i=1}^{6} L_{i}$ with

$$
V=5(1-0.05 \kappa) .
$$

Figure 3.9 shows the profile of the spirals at $t=0,0.5,0.505,0.510,0.515,0.520$, and Figure 3.10 shows the surface at $t=0.520$. In this case we choose $\alpha_{i}=\pi(i-1) / 3$ and $\lambda_{i}=6 / \pi$ for $i=1,2,3,4,5,6$ to construct $u_{0}$ as (3.10). 

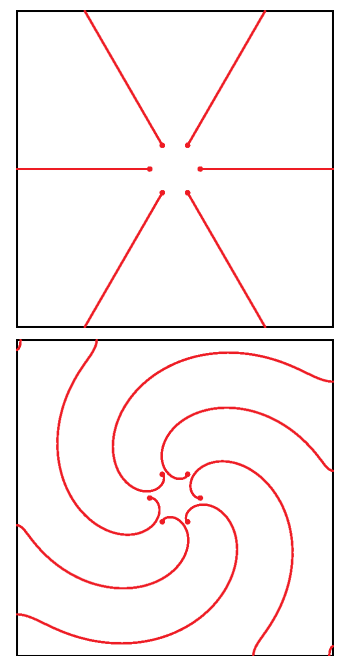
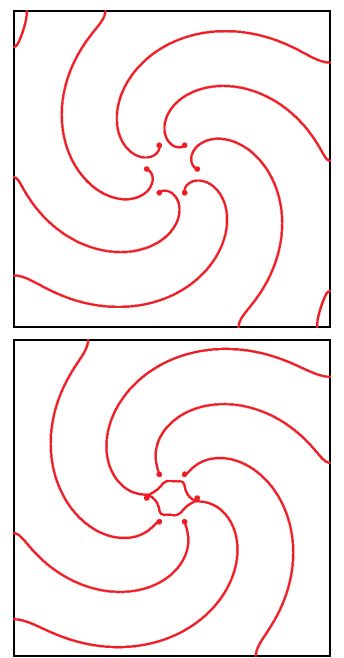
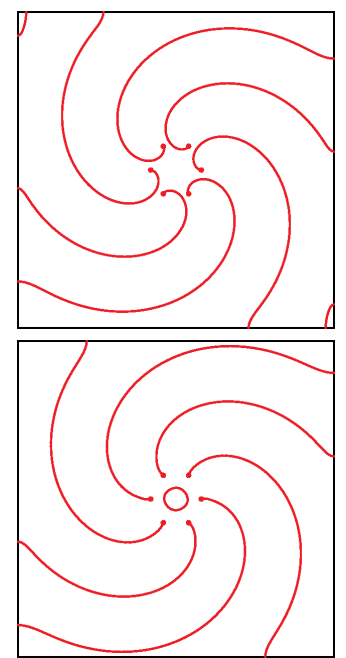

FiguRE 3.9. Evolution of surface by a general group of 6 centers at $t=0$, $0.5,0.505,0.510,0.515,0.520$.

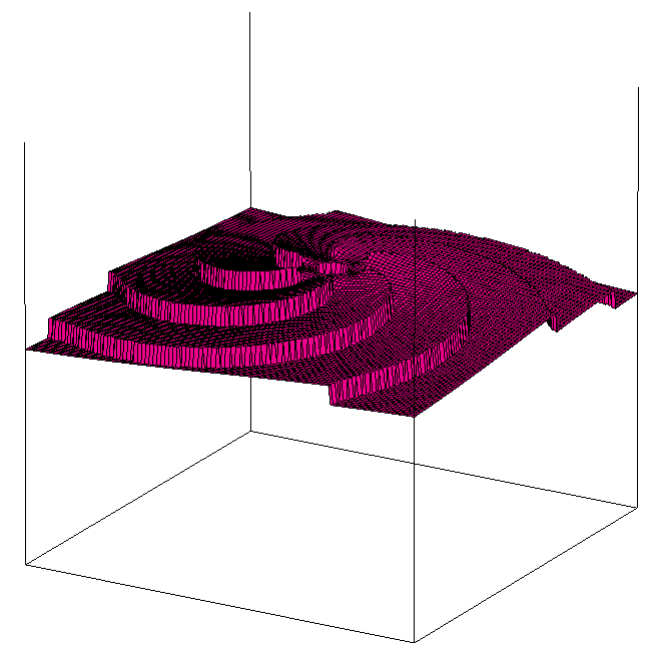

FiguRE 3.10. The surface at $t=0.520$ in figure 3.9.

We note that in the setting prescribed above, the centers satisfy

$$
\left|a_{i+1}-a_{i}\right|= \begin{cases}0.16 & \text { for } i=2,5 \\ 0.17 & \text { otherwise }\end{cases}
$$

and satisfy $\left|a_{i+1}-a_{i}\right|<2 \pi \rho_{c}=\pi / 10$. Therefore these centers are regarded as an effective single center. Actually the profile of spirals in Figure 3.9 is very close to that of single center with six branches. However, one finds a closed curve inside of the group at $t=0.520$. This curve is generated by rotating spirals which touch the centers of their neighboring spirals at some time during the evolution. Thus this curve describes the boundary of a pit in the surface. Because of the driving force and the curvature of the boundary, the 
pit disappears in a short time. However, the height of the surface at where the pit used to be remains lower than the surrounding.

3.4. Pair of screw dislocations with opposite rotational orientations. Consider a pair of spirals with opposite rotational orientations. For simplicity we say that such a pair an opposite pair. This case is described by our formulation with

$$
\theta(x)=m\left(m_{1} \arg \left(x-a_{1}\right)-m_{2} \arg \left(x-a_{2}\right)\right),
$$

where $m_{1}, m_{2} \in \mathbb{N}$ are numbers of spirals associated with $a_{1}$ and $a_{2}$, respectively, and $m \in\{ \pm 1\}$ is a constant defining the rotational orientations, i.e., $m=1$ (resp. $m=-1$ ) if the spirals associated with $a_{1}$ are counter-clockwise (resp. clockwise) and thus those associated with $a_{2}$ are clockwise (resp. counter-clockwise) orientations.

A simple nontrivial example is

$$
\theta(x)=\arg \left(x-a_{1}\right)-\arg \left(x-a_{2}\right)
$$

with $\Gamma_{0}$ as follows

(A) $\Gamma_{0}=\left\{\sigma a_{1}+(1-\sigma) a_{2} \in \bar{W} ; \sigma \in(0,1)\right\}$,

(B) $\Gamma_{0}=L_{1} \cup L_{2}, L_{i}=\left\{a_{i}+r\left(\cos \alpha_{i}, \sin \alpha_{i}\right) \in \bar{W} ; r>0\right\}$ for given constants $\alpha_{1}, \alpha_{2} \in \mathbb{R}$.

Case (A) is already mentioned in $§ 2.4$ and thus we set

$$
u_{0}(x)=\pi \text {. }
$$

Case (B) is similar to the situation discussed in $\S 3.3$. If $\alpha_{1}=\arg \left(a_{1}-a_{2}\right)$ and $\alpha_{2}=$ $\arg \left(a_{2}-a_{1}\right)$ then we set

$$
u_{0}(x)=0 .
$$

Figure 3.11 shows a simulation involving an opposite pair belonging to Case (A), and Figure 3.12 shows the profile of the surface at time $t=0.5$, which is reconstructed from the solution $u$. In the simulation, we set $\theta$ and $u_{0}$ as (3.12) and (3.13), respectively. In Figure 3.10, one sees that the spiral curve changes from an open curve to a closed one, and then to an open curve again; it also splits into different connected pieces when the curve intersects itself. All of these phenomena are computed effortlessly by the proposed method.

To set up a configuration belonging to Case (B), we first set $u_{1}=\alpha_{1}$ and $u_{2}=-\alpha_{2}$ to obtain

$$
\begin{aligned}
& L_{1}=\left\{x \in \bar{W} ; u_{1}(x)-\theta_{1}^{+}(x) \equiv 0 \quad \bmod 2 \pi \mathbb{Z}\right\}, \\
& L_{2}=\left\{x \in \bar{W} ; u_{2}(x)-\theta_{2}^{-}(x) \equiv 0 \quad \bmod 2 \pi \mathbb{Z}\right\}
\end{aligned}
$$

with $\theta_{i}^{ \pm}(x)= \pm \arg \left(x-a_{i}\right)$ for $i=1,2$. We next set

$$
\begin{aligned}
& u_{0}(x)=v_{1}(x)+v_{2}(x)+\pi, \\
& v_{1}(x)=\Theta_{1}^{+}(x)+2 \pi k_{1}(x)+\pi H_{1}\left(\lambda_{1}\left\{u_{1}-\left(\Theta_{1}^{+}(x)+2 \pi k_{1}(x)\right)\right\}\right), \\
& v_{2}(x)=\Theta_{2}^{-}(x)+2 \pi k_{2}(x)+\pi H_{1}\left(\lambda_{2}\left\{u_{2}-\left(\Theta_{2}^{-}(x)+2 \pi k_{2}(x)\right)\right\}\right)
\end{aligned}
$$

as in (3.6), where $\Theta_{i}^{ \pm}$is the principal value of $\theta_{i}^{ \pm}$, i.e., $\pm \arg \left(x-a_{i}\right)$ for $i=1,2$. Here $k_{i}: \bar{W} \rightarrow \mathbb{Z}$ is a function satisfying (3.7) with $u_{i, j}=u_{i}, v_{i, j}=v_{i}, k_{i, j}=k_{i}$ for $i=1,2$. The coefficients $\lambda_{i}$ are constants satisfying eqrefcoprime with $\Lambda_{i, j}=\Lambda_{i}$ for $i=1,2$, i.e., $\Lambda_{1} \cap \Lambda_{2}=\emptyset$. 

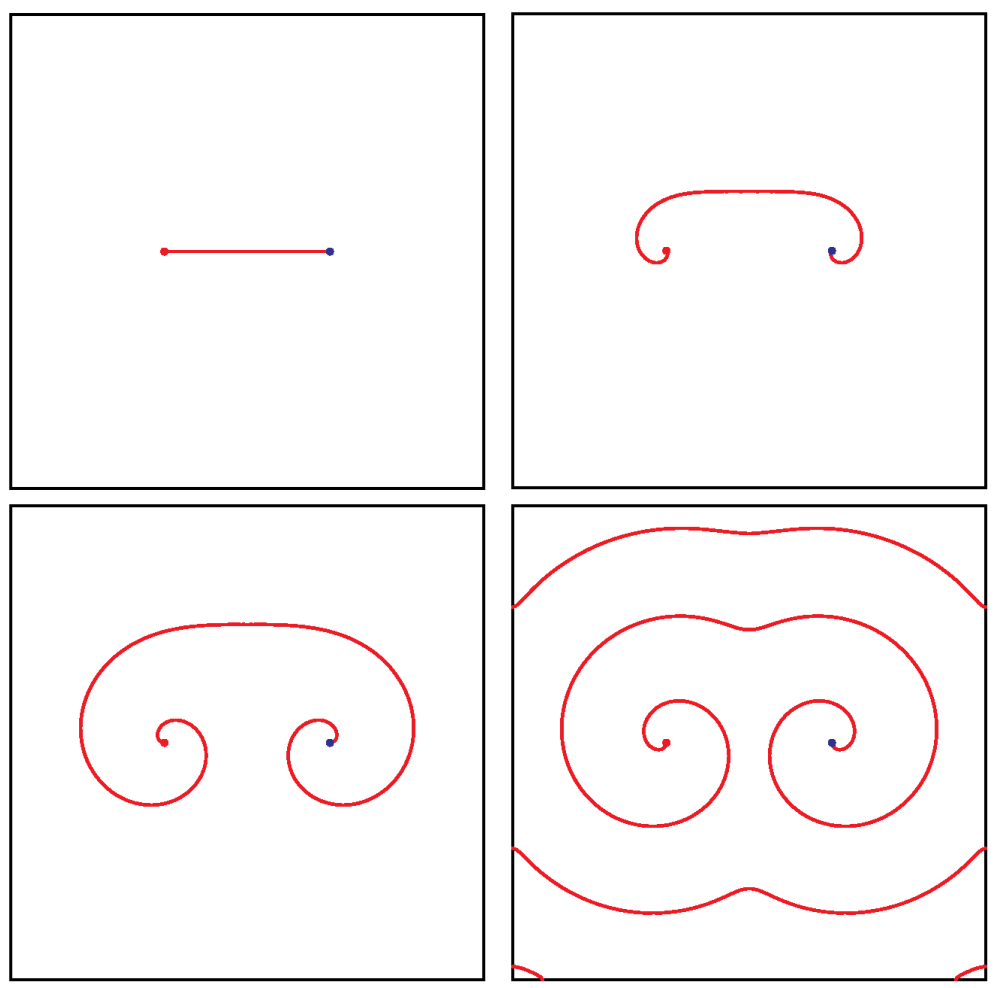

Figure 3.11. The evolution of an opposite pair by (3.5) with initial line (A) at time $t=0, t=0.05, t=0.1, t=0.5$ from left-top to right-bottom.

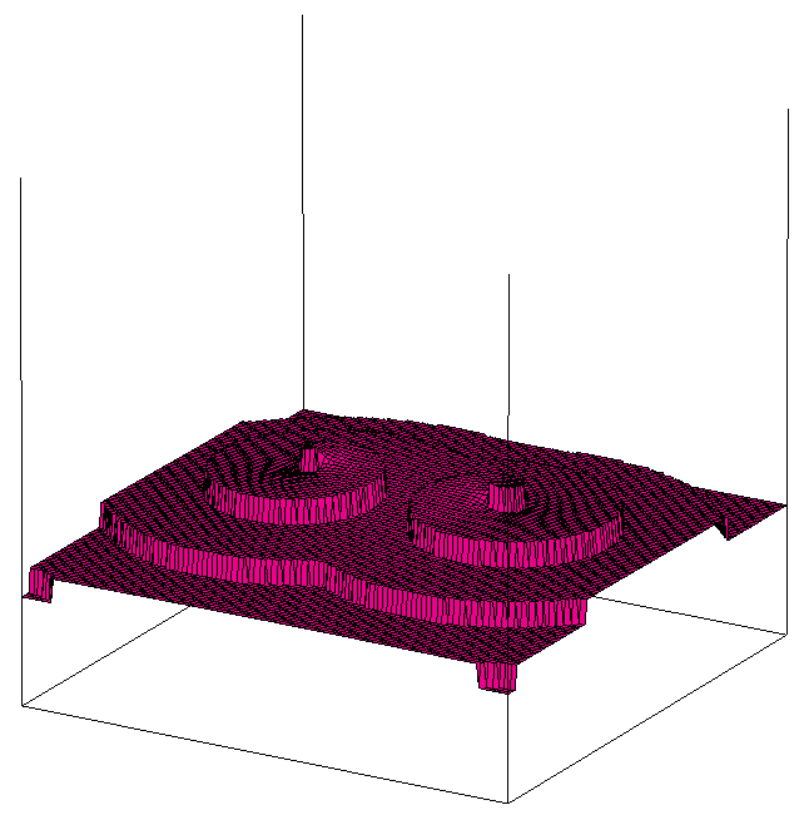

FiguRE 3.12. The profile of the surface at $t=0.5$ from figure 3.11 , which is reconstructed from the numerical solution of the level set equation. 
If an opposite pair $a_{1}$ and $a_{2}$ have $m_{1}$ and $m_{2}$ spirals, respectively, then it is convenient for construction of $u_{0}$ to make groups of simple and connecting spirals for an initial curve. Hence we define $\Gamma_{0}$ by

$$
\begin{aligned}
\Gamma_{0} & =\left(\bigcup_{j=1}^{\tilde{m}_{1}} L_{1, j}\right) \cup\left(\bigcup_{j=1}^{\tilde{m}_{2}} L_{2, j}\right) \cup\left(\bigcup_{n=1}^{n_{c}} L_{c, n}\right), \\
L_{1, j} & =\left\{x \in \bar{W} ; u_{1, j}(x)-\theta_{1}^{+}(x) \equiv 0 \bmod 2 \pi \mathbb{Z}\right\}, \\
L_{2, j} & =\left\{x \in \bar{W} ; u_{2, j}(x)-\theta_{2}^{-}(x) \equiv 0 \bmod 2 \pi \mathbb{Z}\right\}, \\
L_{c, n} & =\left\{x \in \bar{W} ; u_{c, n}(x)-\left(\theta_{1}^{+}(x)+\theta_{2}^{-}(x)\right) \equiv 0 \bmod 2 \pi \mathbb{Z}\right\}
\end{aligned}
$$

with $u_{c, n}, u_{1, j}, u_{2, j} \in C(\bar{W})$. Here $L_{1, j}$ and $L_{2, j}$ denote simple spirals associated with $a_{1}$ and $a_{2}$, respectively, and $L_{c, n}$ denotes connecting spirals, so the numbers $n_{c}, \tilde{m}_{1}$, and $\tilde{m}_{2} \in \mathbb{N}$ of each spirals satisfy $\tilde{m}_{1}+n_{c}=m_{1}$ and $\tilde{m}_{2}+n_{c}=m_{2}$. For connecting spirals $L_{c, n}$ we also introduce modified initial data $v_{c, n}$ and slope sets $\Lambda_{c, n}$, which is similar as (3.6) and (3.9) respectively, of the form

$$
\begin{aligned}
& v_{c, n}(x)=\Theta_{1}^{+}(x)+\Theta_{2}^{-}(x)+2 \pi k_{c, n}(x) \\
&+\pi H_{1}\left(\lambda_{c, n}\left\{u_{c, n}-\left(\Theta_{1}^{+}(x)+\Theta_{2}^{-}(x)+2 \pi k_{c, n}(x)\right)\right\}\right), \\
& \Lambda_{c, n}=\left\{x \in \bar{W} ;\left|v_{c, n}(x)-\left(\Theta_{1}^{+}(x)+\Theta_{2}^{-}(x)+2 \pi k_{c, n}(x)\right)\right|<\pi\right\},
\end{aligned}
$$

where $\lambda_{c, n}>1 / \pi$ is a constant and $k_{c, n}: \bar{W} \rightarrow \mathbb{Z}$ is such that

$$
-\pi \leq u_{c, n}(x)-\left(\Theta_{1}^{+}(x)+\Theta_{2}^{-}(x)+2 \pi k_{c, n}(x)\right)<\pi \quad \text { for } x \in \bar{W} .
$$

For construction of initial data $u_{0} \in C(\bar{W})$ similarly as (3.10) we choose $\lambda_{1, j}, \lambda_{2, j}$ and $\lambda_{c, n}$ such that

$$
\Lambda_{c, n} \cap \Lambda_{i, j}=\emptyset \quad \Lambda_{c, n_{1}} \cap \Lambda_{c, n_{2}}=\emptyset \quad \text { if } n_{1} \neq n_{2},
$$

in addition to $(3.8)$ for $n, n_{1}, n_{2}$ and $(i, j)$. Then, we set

$$
u_{0}(x)=\sum_{n=1}^{n_{c}} v_{c, n}(x)+\sum_{j=1}^{\tilde{m}_{1}} v_{1, j}(x)+\sum_{j=1}^{\tilde{m}_{2}} \tilde{v}_{2, j}(x)+\left(n_{c}+\tilde{m}_{1}+\tilde{m}_{2}-1\right) \pi
$$

and obtain

$$
\Gamma_{0}=\left\{x \in \bar{W} ; u_{0}(x)-\theta(x) \equiv 0 \quad \bmod 2 \pi \mathbb{Z}\right\}
$$

with $\theta(x)=m_{1} \arg \left(x-a_{1}\right)-m_{2} \arg \left(x-a_{2}\right)$. If we consider the opposite rotational orientations of the above, then we change $a_{1}$ and $a_{2}$ and do above.

We have two examples of simulations. The first one is for the same initial curve as figure 3.2, but $L_{2,1}, L_{2,2}$ have the clockwise orientations (see figure 3.13). In this case we set

and set

$$
u_{1,1} \equiv \pi, \quad u_{2,1} \equiv-\frac{\pi}{3}, \quad u_{2,2} \equiv \frac{\pi}{3}, \quad \lambda_{1,1}=\lambda_{2,1}=\lambda_{2,2}=\frac{3}{\pi}
$$

$$
u_{0}(x)=v_{1,1}(x)+v_{2,1}(x)+v_{2,2}(x)+2 \pi .
$$



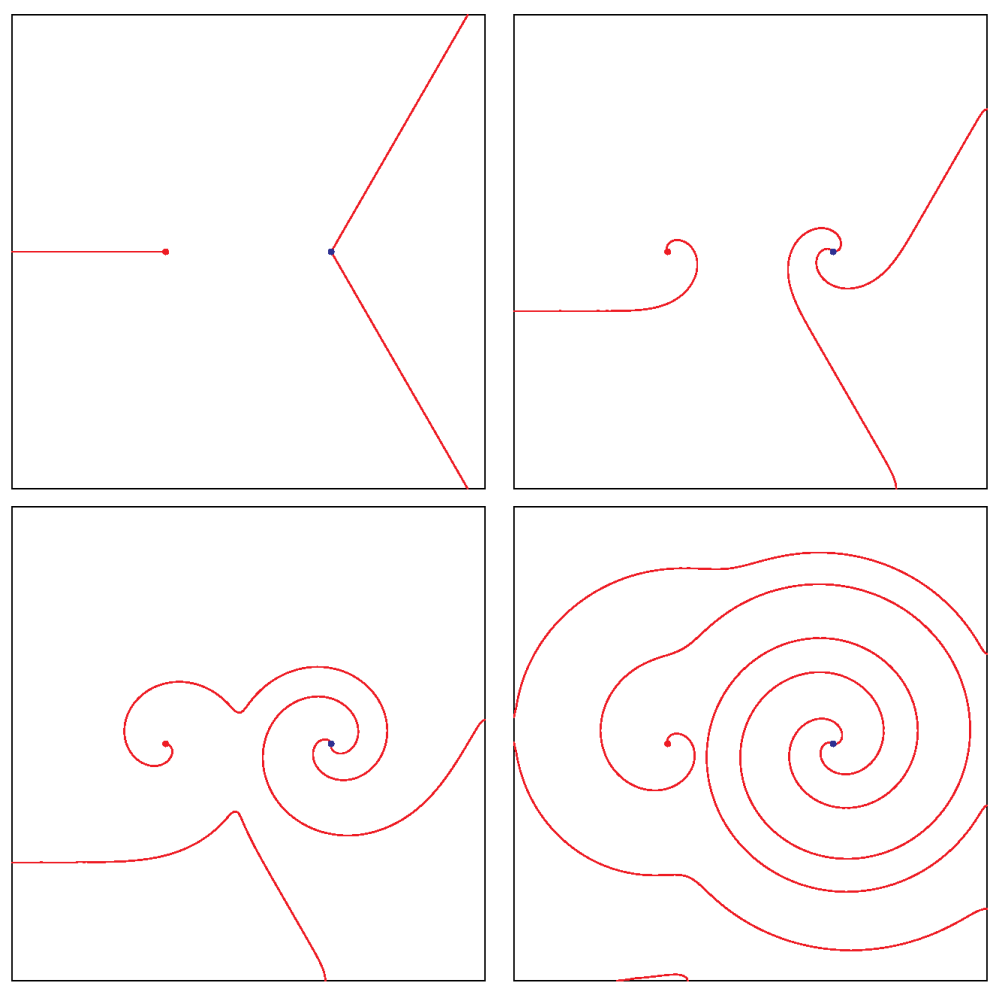

FiguRE 3.13. Simulation of single spirals associated with $a_{1}=(-0.35,0)$ with the counter-clockwise orientations, and two spirals associated with $a_{2}=(0.35,0)$ with clockwise orientations. The evolution equation is $(3.5)$, i.e., $v_{\infty}=5$ and $\rho_{c}=0.02$. The above figures are profiles of spirals at $t=0,0.05,0.1$ and 0.2 from left top to right bottom.

The second one is by a connecting lines and a simple spiral line associated with $a_{2}$, i.e.,

$$
\begin{aligned}
\Gamma_{0} & =L_{c} \cup L_{2}, \\
L_{c} & =\left\{\sigma a_{1}+(1-\sigma) a_{2} \in \bar{W} ; \sigma \in(0,1)\right\}, \\
L_{2} & =\left\{a_{2}+(r, 0) \in \bar{W} ; r>0\right\} .
\end{aligned}
$$

(See figure 3.14.) In this case we set $u_{c} \equiv \pi$ and $u_{2} \equiv 0$ to obtain

$$
\begin{aligned}
& L_{c}=\left\{x \in \bar{W} ; u_{c}(x)-\left(\theta_{1}^{+}(x)+\theta_{2}^{-}(x)\right) \equiv 0 \bmod 2 \pi \mathbb{Z}\right\}, \\
& L_{2}=\left\{x \in \bar{W} ; u_{2}(x)-\theta_{2}^{-}(x) \equiv 0 \bmod 2 \pi \mathbb{Z}\right\},
\end{aligned}
$$

and set

with $\lambda_{c}=\lambda_{c, 1}=\pi / 2$ and $\lambda_{2}=\lambda_{2,1}=\pi / 2$.

$$
u_{0}(x)=v_{c}(x)+v_{2}(x)+\pi
$$

Remark 3.4. Burton et al [BCF51, 9, Appendix B] give an interesting observation on the growth of a crystal surface by an opposite pair $a_{1}$ and $a_{2}$ :

(i) $\left|a_{1}-a_{2}\right|<2 \rho_{c}$ : the pair have no influence on the growth of the surface (they call this pair an inactive pair); 

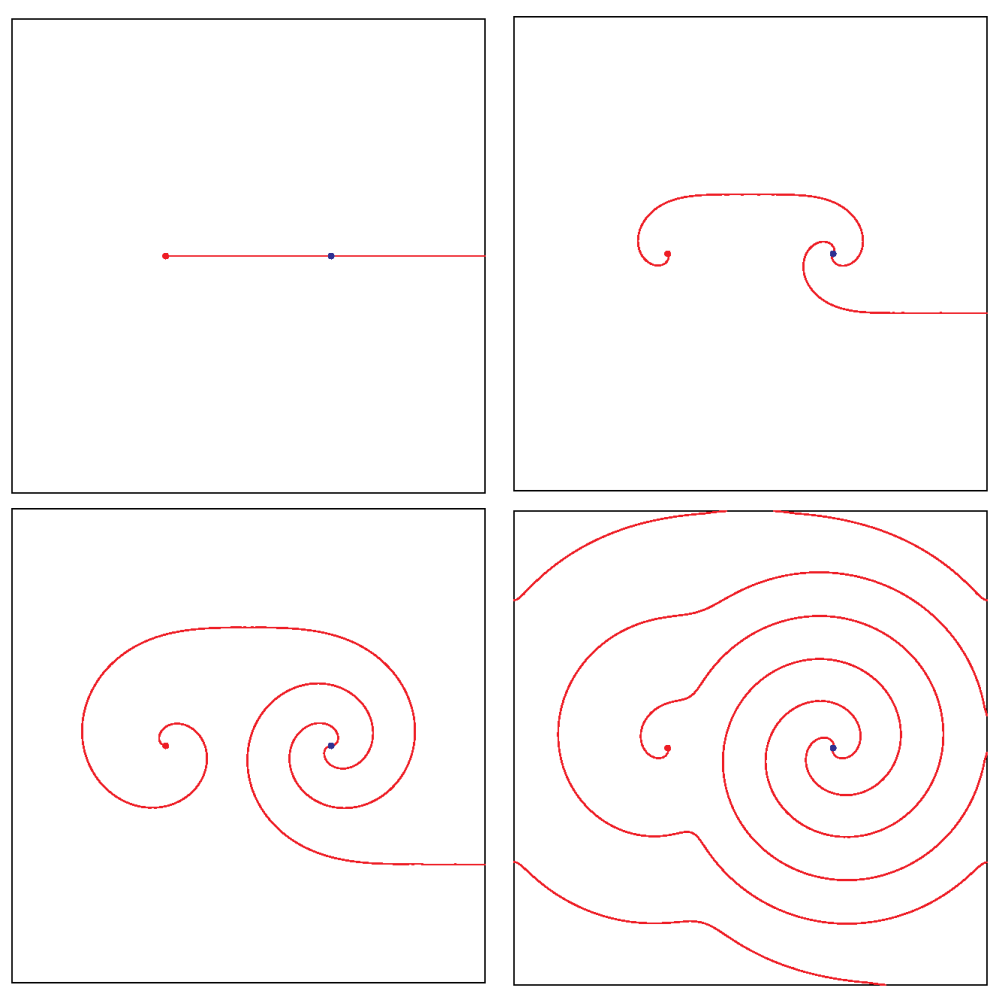

FiguRE 3.14. Simulation of similar case as figure 3.13 started from single connecting line and single simple line associated with $a_{2}$. The equation, and times of each profiles are same as figure 3.13.

(ii) $\left|a_{1}-a_{2}\right|<3 \rho_{c}$ : the growth rate of the surface is monotonically increasing with respect to the distance of the pair, and become larger than the case of single spirals;

(iii) $\left|a_{1}-a_{2}\right|$ is sufficiently large: the growth rate of the surface decreases with respect to $\left|a_{1}-a_{2}\right|$, and converges to the single one exponentially fast as $\left|a_{1}-a_{2}\right| \rightarrow \infty$.

The proposed method may be used to discover the existence of an inactive pair, the relation between the distance of the pair and the growth rate. From the second results on the above we observe that the growth rate attains its maximum when the distance of the pair is around $4 \rho_{c}$. In [OTG] and [GOT] we prove rigorously the existence of an inactive pair and of curves which play the role of upper bound on the evolution of steps.

3.5. Coarsening. According to Remark 3.4, if there exist several opposite pairs on a surface, then the growth resulting from the closest pair would dominate so that the surface forms one large mountain that peaks around the closest pair. Schulze and Kohn [SK99] approximate this phenomenon by proposing a Hamilton-Jacobi equation with discontinuous source terms at points of dislocations. For a rigorous treatment of such Hamilton-Jacobi equation see a recent work by Hamamuki and the third author [GH11].

Figure 3.15 shows the evolution of two opposite pairs and the emergence of a mountain peaking near the pair on the upper left corner of the domain. The parameters in the 

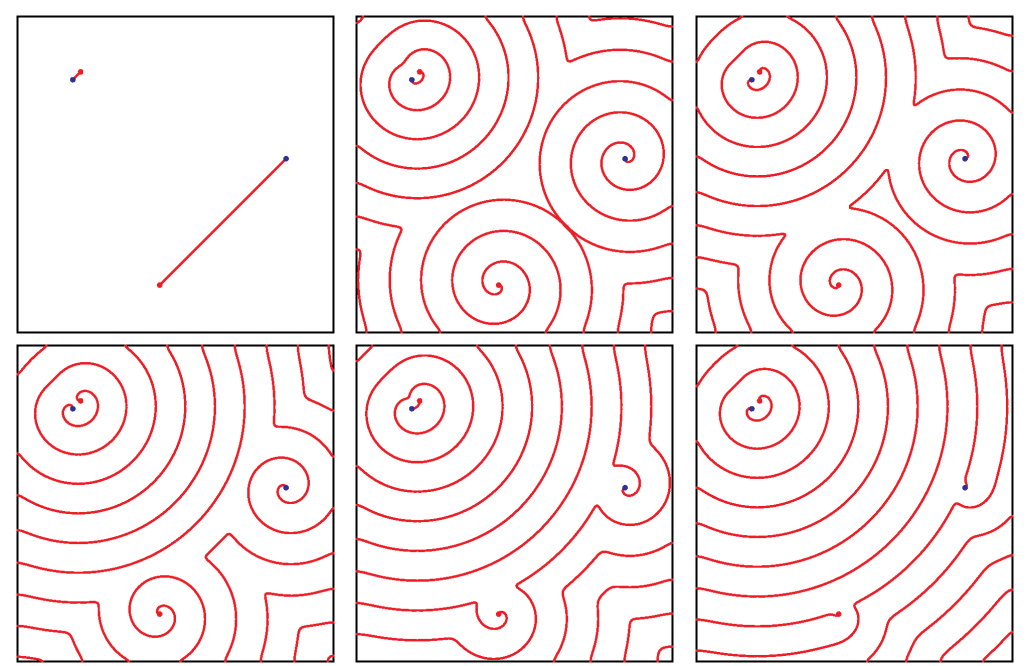

FiguRE 3.15. Evolution of spirals by close and far opposite pairs. The figures are profiles of spirals at $t=0,0.25,0.5,0.75,1.0$ and 1.5.

evolution equation, the center locations, and $\theta$ used in the simulation are given by

$$
\begin{aligned}
& V=20(1-0.01 \kappa) \quad\left(v_{\infty}=20, \rho_{c}=0.01\right), \\
& a_{1}=(-0.65,0.6), a_{2}=(-0.6,0.65), a_{3}=(-0.1,-0.7), a_{4}=(0.7,0.1), \\
& \theta(x)=-\arg \left(x-a_{1}\right)+\arg \left(x-a_{2}\right)+\arg \left(x-a_{3}\right)-\arg \left(x-a_{4}\right) .
\end{aligned}
$$

In constructing the initial data we first set

$$
\begin{aligned}
& u_{1}=u_{2}=\pi, \\
& \theta_{1}(x)=-\arg \left(x-a_{1}\right)+\arg \left(x-a_{2}\right), \theta_{2}(x)=\arg \left(x-a_{3}\right)-\arg \left(x-a_{4}\right)
\end{aligned}
$$

to obtain

$$
\begin{aligned}
L_{1} & =\left\{\sigma a_{1}+(1-\sigma) a_{2} \in \bar{W} ; \sigma \in(0,1)\right\} \\
& =\left\{x \in \bar{W} ; u_{1}(x)-\theta_{1}(x) \equiv 0 \bmod 2 \pi \mathbb{Z}\right\}, \\
L_{2} & =\left\{\sigma a_{3}+(1-\sigma) a_{4} \in \bar{W} ; \sigma \in(0,1)\right\} \\
& =\left\{x \in \bar{W} ; u_{2}(x)-\theta_{2}(x) \equiv 0 \bmod 2 \pi \mathbb{Z}\right\} .
\end{aligned}
$$

Next we modify $u_{1}$ and $u_{2}$ to obtain $u_{0}$ in a similar fashion as in (3.14). We set

$$
v_{i}(x)=\Theta_{i}(x)+2 \pi k_{i}(x)+\pi H_{1}\left(\lambda_{i}\left[u_{i}(x)-\left(\Theta_{i}(x)+2 \pi k_{i}(x)\right)\right]\right),
$$

where $\Theta_{i}$ is a smooth branch of $\theta_{i}$, and $k_{i}: \bar{W} \rightarrow \mathbb{Z}$ is similar as in (3.14). In this simulation we choose $\lambda_{i}=3 /(2 \pi)$ to obtain

$$
\bigcap_{i=1}^{2}\left\{x \in \bar{W} ;\left|v_{i}(x)-\left(\Theta_{i}(x)+2 \pi k_{i}(x)\right)\right|<\pi\right\}=\emptyset,
$$

and set $u_{0}(x)=v_{1}(x)+v_{2}(x)+\pi$. In the simulation, we find annual ring around $a_{1}$ and $a_{2}$, and spirals around $a_{3}$ and $a_{4}$ at $t=0.25$ in Figure 3.15. We observe that the annual rings dominate the surface as the time evolves (see the profile at $t=1.5$ Figure 3.15). 
3.6. More general situation. In this subsection, we examine a more complex situation in which the number and the rotational orientation of the spirals connecting to each screw dislocation are independently given. Figure 3.16 shows the evolution of a set of spirals connecting to six screw dislocations. The evolution equation, location of centers, and $\theta$ is as follows;

$$
\begin{aligned}
& V=5(1-0.03 \kappa) \quad\left(v_{\infty}=5, \rho_{c}=0.03\right), \\
& a_{1}=(-0.6,-0.5), a_{2}=(-0.4,0.2), a_{3}=(-0.2,0.5) \text {, } \\
& a_{4}=(0,-0.4), a_{5}=(0.2,0), a_{6}=(0.4,0.5) \text {, } \\
& \theta(x)=-\arg \left(x-a_{1}\right)+3 \arg \left(x-a_{2}\right)+2 \arg \left(x-a_{3}\right) \\
& +\arg \left(x-a_{4}\right)-3 \arg \left(x-a_{5}\right)-2 \arg \left(x-a_{6}\right) .
\end{aligned}
$$

In this case all spirals associated with $a_{2}, a_{3}$ and $a_{4}$ have the counter-clockwise orientations, those of $a_{1}, a_{5}$ and $a_{6}$ have the clockwise orientations, and $a_{1}, a_{2}, \ldots, a_{6}$ have $1,3,2,1,3$, and 2 spirals, respectively. The initial curve is given as $\Gamma_{0}=\bigcup_{j=1}^{7} L_{j}$, and

$$
\begin{aligned}
& L_{1}=\left\{t a_{1}+(1-t) a_{2} ; t \in(0,1)\right\}, \\
& L_{2}=\left\{a_{2}+t(-1,0) ; t>0\right\}, \\
& L_{3}=\left\{t a_{2}+(1-t) a_{5} ; t \in(0,1)\right\}, \\
& L_{4}=\left\{t a_{3}+(1-t) a_{5} ; t \in(0,1)\right\}, \\
& L_{5}=\left\{t a_{4}+(1-t) a_{5} ; t \in(0,1)\right\}, \\
& L_{6}=\left\{t a_{3}+(1-t) a_{6} ; t \in(0,1)\right\}, \\
& L_{7}=\left\{a_{6}+t(1,0) ; t>0\right\} .
\end{aligned}
$$

To construct $u_{0} \in C(\bar{W})$ satisfying $\Gamma_{0}=\left\{x ; u_{0}(x)-\theta(x) \equiv 0 \bmod 2 \pi \mathbb{Z}\right\}$ we describe $L_{j}=\left\{x \in \bar{W} ; u_{j}(x)-\theta_{j}(x) \equiv 0 \bmod 2 \pi \mathbb{Z}\right\}$ with

$$
\begin{aligned}
& u_{1}=u_{2}=u_{3}=u_{4}=u_{5}=u_{6} \equiv \pi, u_{7} \equiv 0, \\
& \theta_{1}(x)=-\arg \left(x-a_{1}\right)+\arg \left(x-a_{2}\right), \\
& \theta_{2}(x)=\arg \left(x-a_{2}\right), \\
& \theta_{3}(x)=\arg \left(x-a_{2}\right)-\arg \left(x-a_{5}\right), \\
& \theta_{4}(x)=\arg \left(x-a_{3}\right)-\arg \left(x-a_{5}\right), \\
& \theta_{5}(x)=\arg \left(x-a_{4}\right)-\arg \left(x-a_{5}\right), \\
& \theta_{6}(x)=\arg \left(x-a_{3}\right)-\arg \left(x-a_{6}\right), \\
& \theta_{7}(x)=-\arg \left(x-a_{6}\right) .
\end{aligned}
$$

We next construct a modified initial data $v_{i}$ from $u_{i}$ and introduce a slope set $\Lambda_{i}$ similarly as in previous sections, i.e., set

$$
\begin{aligned}
v_{i}(x) & =\Theta_{i}(x)+2 \pi k_{i}(x)+\pi H_{1}\left(\lambda_{i}\left\{u_{i}(x)-\left(\Theta_{i}(x)+2 \pi k_{i}(x)\right)\right\}\right) \\
\Lambda_{i} & =\left\{x \in \bar{W} ;\left|v_{i}(x)-\left(\Theta_{i}(x)+2 \pi k_{i}(x)\right)\right|<\pi\right\},
\end{aligned}
$$

where $\Theta_{i}$ is a smooth branch of $\theta_{i}, k_{i}: \bar{W} \rightarrow \mathbb{Z}$ is such that

$$
-\pi \leq u_{i}(x)-\left(\Theta_{i}(x)+2 \pi k_{i}(x)\right)<\pi \quad \text { for } x \in \bar{W},
$$



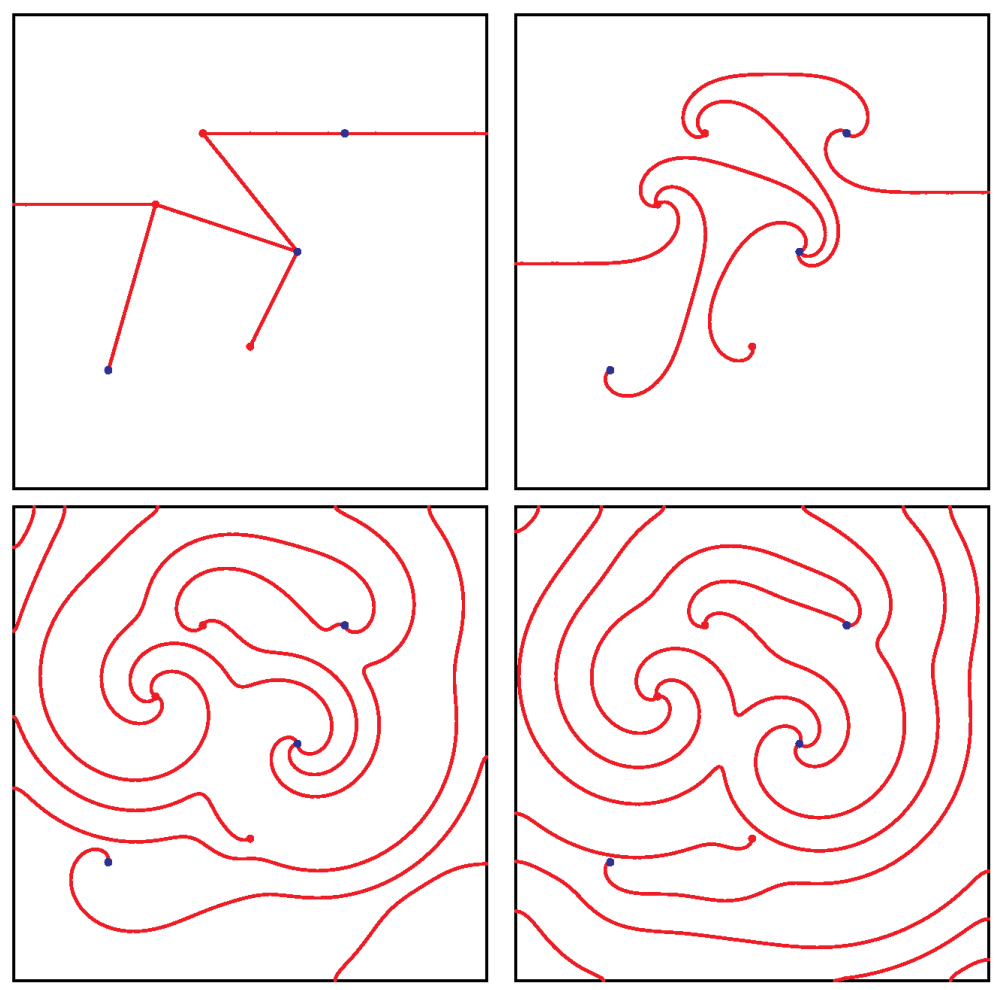

Figure 3.16. Evolution of multiple spirals connecting to six centers with multiple spirals by $V=5(1-0.03 \kappa)$. Left top, right top, left bottom and right bottom figures are level sets at time $t=0,0.05,0.2$, and 0.5 , respectively.

and $\lambda_{i}>1 / \pi$ is a constant satisfying $\Lambda_{i} \cap \Lambda_{j}=\emptyset$ for $i, j$ provided that $i \neq j$. For calculation in figure 3.16 we choose

$$
\lambda_{1}=\lambda_{2}=\frac{5}{\pi}, \lambda_{3}=\lambda_{4}=\frac{10}{\pi}, \lambda_{5}=\lambda_{6}=\lambda_{7}=\frac{3}{\pi} .
$$

We set the initial data as

$$
u_{0}(x)=\sum_{i=1}^{7} v_{i}(x)+6 \pi .
$$

In this simulation, the surface around $a_{2}$ and $a_{4}$ grows the fastest. It is due to the fact that the surface near a screw dislocation with $m$ spirals evolves (grows) with $m$ times the rate of that near a screw dislocation with a single spiral.

\section{Conclusion}

We have introduced a flexible level set formulation for modeling multiple spirals that possibly have different rotational orientations. Our formulation embeds a set of spirals as the zero level set of the difference of an explicitly defined sheet structure function and an auxiliary function, which is computed numerically. As the first author [Oht03] or Goto, Nakagawa and the first author [GNO08] studied, our model has the potential to verify the dynamical behavior of spirals rigorously. 
The crucial idea of our formulation comes from a sheet structure function due to Kobayashi [Kob10]. The sheet structure function is a linear combination of arguments with respect to the centers of screw dislocations in the domain. Our formulation had been studied in [Oht03] or [GNO08], however there were no explanations on the coefficients from physical view points in those papers. In this paper, we clarify how the coefficients in the linear combination are determined from a given physical configuration. We also give a simple and practical way to construct initial auxiliary functions.

Our formulation requires only a single equation model for evolution of spirals by (1.1)(1.2). In this regard, our formulation is more computationally tractable. We have verified the results in Burton et al [BCF51] as well as in [Sme00]. Furthermore, we presented our simulations involving multi-centers and multi-spirals configurations and non-trivial merging. Such situations seem to pose computational challenges for other approaches, including the one proposed in[Sme00]. We point out here that in a forthcoming paper [GOT], we shall discuss the existence of what we called inactive pairs (pairs of stationary spirals) and show analytically the stability of bunched steps.

Our model can easily be generalized to describe anisotropic evolution of spirals, and thus it can describe the evolution with interlacing patterns. Finally, our formulation has the potential to be generalized to model moving or nucleation of spiral centers. From the view point of physical experiments it is required to construct a system which implies an evolution or a flow of the concentration of atoms on the surface or in environment phase. To know the exact mechanism of generation of hollow cores we have to construct a formulation of spirals with tip motion. To adjoin our method to the above situations, we need additional modelings. (See [SB82] for an interlacing pattern or a hollow core.)

\section{REFERENCES}

[BCF51] W. K. Burton, N. Cabrera, and F. C. Frank. The growth of crystals and the equilibrium structure of their surfaces. Philosophical Transactions of the Royal Society of London. Series A. Mathematical and Physical Sciences, 243:299-358, 1951.

[CGG91] Yun Gang Chen, Yoshikazu Giga, and Shun'ichi Goto. Uniqueness and existence of viscosity solutions of generalized mean curvature flow equations. J. Differential Geom., 33(3):749-786, 1991.

[CIL92] Michael G. Crandall, Hitoshi Ishii, and Pierre-Louis Lions. User's guide to viscosity solutions of second order partial differential equations. Bull. Amer. Math. Soc. (N.S.), 27(1):1-67, 1992.

[CT08] Li-Tien Cheng and Yen-Hsi Tsai. Redistancing by flow of time dependent eikonal equation. J. Comput. Phys., 227(8):4002-4017, 2008.

[ES91] L. C. Evans and J. Spruck. Motion of level sets by mean curvature. I. J. Differential Geom., 33(3):635-681, 1991.

[FGT04] Bernold Fiedler, Jong-Shenq Guo, and Je-Chiang Tsai. Multiplicity of rotating spirals under curvature flows with normal tip motion. J. Differential Equations, 205(1):211-228, 2004.

[FGT06] Bernold Fiedler, Jong-Shenq Guo, and Je-Chiang Tsai. Rotating spirals of curvature flows: a center manifold approach. Ann. Mat. Pura Appl. (4), 185(suppl.):S259-S291, 2006.

[FIM] N. Forcadel, C. Imbert, and R. Monneau. Uniqueness and existence of spirals moving by forced mean curvature motion. preprint.

[GH11] Y. Giga and N. Hamamuki. Hamilton-jacobi equations with discontinuous source terms. Hokkaido University Preprint series \#987, 2011. to appear in Comm. Partial Differential Equations.

[Gig06] Yoshikazu Giga. Surface evolution equations: A level set approach, volume 99 of Monographs in Mathematics. Birkhäuser Verlag, Basel, 2006. 
[GIK02] Yoshikazu Giga, Naoyuki Ishimura, and Yoshihito Kohsaka. Spiral solutions for a weakly anisotropic curvature flow equation. Adv. Math. Sci. Appl., 12(1):393-408, 2002.

[GNO08] Shun'ichi Goto, Maki Nakagawa, and Takeshi Ohtsuka. Uniqueness and existence of generalized motion for spiral crystal growth. Indiana University Mathematics Journal, 57(5):2571$2599,2008$.

[GNOT06] Jong-Shenq Guo, Ken-Ichi Nakamura, Toshiko Ogiwara, and Je-Chiang Tsai. On the steadily rotating spirals. Japan J. Indust. Appl. Math., 23(1):1-19, 2006.

[GOT] Yoshikazu Giga, Takeshi Ohtsuka, and Yen-Hsi Richard Tsai. Inactive pairs and stability of bunched curves for spiral evolution by eikonal-curvature flow equations (in preparation).

[HL68] John Price Hirth and Jens Lothe. Theory of dislocations. McGraw-Hill Education, New York, 1968.

[IIU99] Hitoshi Imai, Naoyuki Ishimura, and TaKeo Ushijima. Motion of spirals by crystalline curvature. M2AN Math. Model. Numer. Anal., 33(4):797-806, 1999.

[Ish98] Naoyuki Ishimura. Shape of spirals. Tohoku Math. J. (2), 50(2):197-202, 1998.

[Kob10] Ryo Kobayashi. A brief introduction to phase field method. AIP Conf. Proc., 1270:282-291, 2010.

[KP98] Alain Karma and Mathis Plapp. Spiral surface growth without desorption. Phys. Rev. Lett., 81:4444-4447, Nov 1998.

[OF01] Stanley Osher and Ronald P. Fedkiw. Level set methods: an overview and some recent results. J. Comput. Phys., 169(2):463-502, 2001.

[Oht03] Takeshi Ohtsuka. A level set method for spiral crystal growth. Advances in Mathematical Sciences and Applications, 13(1):225-248, 2003.

[ON03] Toshiko Ogiwara and Ken-Ichi Nakamura. Spiral traveling wave solutions of nonlinear diffusion equations related to a model of spiral crystal growth. Publ. Res. Inst. Math. Sci., 39(4):767-783, 2003.

[OS88] Stanley Osher and James A. Sethian. Fronts propagating with curvature-dependent speed: algorithms based on Hamilton-Jacobi formulations. J. Comput. Phys., 79(1):12-49, 1988.

[OTG] Takeshi Ohtsuka, Yen-Hsi Richard Tsai, and Yoshikazu Giga. On the growth rate of a crystal surface with several dislocation centers (in preparation).

[PW67] Murray H. Protter and Hans F. Weinberger. Maximum principles in differential equations. Prentice-Hall Inc., Englewood Cliffs, N.J., 1967.

[SB82] I. Sunagawa and P. Bennema. Morphology of growth spirals: Theoretical and experimental. Preparation and Properties of Solid State Materials, Growth mechanisms and silicon nitride, 7:1-129, 1982.

[Set99] J. A. Sethian. Level set methods and fast marching methods. Cambridge University Press, Cambridge, second edition, 1999. Evolving interfaces in computational geometry, fluid mechanics, computer vision, and materials science.

[SK99] T. P. Schulze and R. V. Kohn. A geometric model for coarsening during spiral-mode growth of thin films. Physica D. Nonlinear Phenomena, 132(4):520-542, 1999.

[Sme00] Peter Smereka. Spiral crystal growth. Physica D. Nonlinear Phenomena, 138(3-4):282-301, 2000.

[TO05] Yen-Hsi Richard Tsai and Stanley Osher. Total variation and level set methods in image science. Acta Numer., 14:509-573, 2005.

[XCSE03] Y Xiang, LT Cheng, DJ Srolovitz, and WN E. A level set method for dislocation dynamics. Acta Materialia, 51(18):5499-5518, 2003.

[XSCW04] Y Xiang, DJ Srolovitz, LT Cheng, and E Weinan. Level set simulations of dislocation-particle bypass mechanisms. Acta Materialia, 52(7):1745-1760, 2004. 
Division of Mathematical Sciences, Graduate School of Engineering, Gunma University, 4-2 Aramaki-Machi, Maebashi-Shi, Gunma 371-8510, Japan

E-mail address: tohtsuka@gunma-u.ac.jp

Department of Mathematics and Institute for Computational Engineering and Sciences(ICES), The University of Texas at Austin, TeXas 78712

Graduate School of Mathematical Sciences, University of Tokyo, Komaba 3-8-1, MeguroKU, TOKYO 153-8914, JAPAN

E-mail address: labgiga@ms.u-tokyo.ac.jp 